\title{
Molecular gas associated with RCW 121 and RCW 122
}

\author{
E. M. Arnal ${ }^{1,2, \star}$, N. U. Duronea ${ }^{1, \star \star}$, and J. C. Testori ${ }^{1, \star \star \star}$ \\ 1 Instituto Argentino de Radioastronomía (CCT-La Plata, CONICET), C.C. No. 5, 1894 Villa Elisa, Argentina \\ e-mail: arnal@iar.unlp.edu.ar \\ ${ }^{2}$ Facultad de Ciencias Astronómicas y Geofísicas, Universidad Nacional de La Plata, Paseo del Bosque s/n, 1900 La Plata, Argentina
}

Received 22 November 2007 / Accepted 30 April 2008

\section{ABSTRACT}

\begin{abstract}
Aims. We analyse the distribution of the molecular gas towards the region containing the open cluster Havlen-Moffat 1 (HM 1) the Wolf-Rayet stars WR 87, WR 89, and WR 91, and the star forming regions RCW 121 and RCW 122, with the aim of looking for a possible physical relationship among these objects.

Methods. We used the carbon monoxide observations carried out at $\lambda \sim 2.6 \mathrm{~mm}$ with the $4 \mathrm{~m}$ NANTEN radiotelescope; new flux density determinations derived from already existing radio continuum surveys at $2.417,5,8.35$, and $14.35 \mathrm{GHz}$; continuum flux density determinations available in the literature; and the Midcourse Space Experiment (MSX) and the Improved Reprocessing of the IRAS Survey (IRIS) databases.

Results. Adopting a distance of $5 \mathrm{kpc}$ for RCW 121 and RCW 122, we found a giant molecular cloud (GMC) with a linear extent of $\sim 100 \times 20 \mathrm{pc}$ to be associated with galactic star-forming regions. The total mass of this GMC is of the order of $1.2 \times 10^{6}$ solar masses and its mean radial velocity is about $-15 \mathrm{~km} \mathrm{~s}^{-1}$. Within the GMC there are individual molecular gas concentrations, having total molecular masses in the range from $4.6 \times 10^{4} M_{\odot}$ (RCW 122 C) to $2.2 \times 10^{5} M_{\odot}(\mathrm{RCW} 122)$. The CO profiles observed toward the peak of the molecular concentrations are broad, with typical full-width half-maximum around 6 to $7 \mathrm{~km} \mathrm{~s}^{-1}$, and show line asymmetries and/or double-peaked shape that change with the observed position within a given $\mathrm{CO}$ concentration. An analysis of the MSX and IRAS databases show that each CO concentration has a strong IR counterpart. The dust temperature of these concentrations range from $46 \mathrm{~K}(\mathrm{RCW} 121)$ to $76 \mathrm{~K}(\mathrm{RCW} 122 \mathrm{C})$. Their infrared luminosity are a few times $10^{5} \mathrm{~L}_{\odot}$. The new radio continuum flux density determinations are in good agreement with previous determinations at other frequencies, and confirm the thermal nature of RCW 121 and RCW 122. Based on the newly-determined $5 \mathrm{GHz}$ flux density, we found that to power these HII regions, each of them must harbour a sizable number of $\mathrm{O}$ type stars. Under the assumption that all the ionizing stars have a O7 V spectral type, at least $\sim 8$ and $\sim 4$ of these stars would be needed to ionize RCW 122 and RCW 121, respectively.
\end{abstract}

Key words. ISM: molecules - radio continuum: ISM - infrared: ISM - ISM: HII regions - ISM: individual object: RCW 121 ISM: individual object: RCW 122

\section{Introduction}

It is widely accepted that massive stars are born deeply buried within dense molecular clouds that prevent them from being observed at optical wavelengths. Fortunately, the strong UV radiation field emitted by a massive star dissociates and ionizes the surrounding gas, forming a compact HII region that could be observed at radio wavelengths. Moreover, a sizable fraction of the stellar photons will also heat the dust located nearby, which will eventually radiate in the far-infrared (FIR).

Star forming regions $\mathrm{RCW} 121\left[(l, b)=\left(348^{\circ} .24,-00^{\circ} .98\right)\right]$ and $\operatorname{RCW} 122\left[(l, b)=\left(348^{\circ} .73,-1^{\circ} .04\right)\right]$ were first noticed in the catalogue of $\mathrm{H} \alpha$-emission regions in the Southern Milky Way (Rodgers et al. 1960). Both objects are described as small patches of medium $\mathrm{H} \alpha$ surface brightness.

The open cluster Havlen-Moffat 1 (HM 1) is located about $20^{\prime}$ northwards of RCW 122. This cluster, whose angular apparent diameter is $\sim 5$ arcmin (Dias et al. 2002), harbours a very compact group of $\mathrm{O}_{f}$ stars (Sanduleak 1974) and is placed at a distance of 2900 pc (Mermilliod 1999). The

^ Member of Carrera del Investigador Científico, CONICET, Argentina.

$\star \star$ Fellow of CONICET, Argentina.

$\star \star \star$ Member of Carrera del Personal de Apoyo, CONICET, Argentina.
Wolf-Rayet (WR) stars WR $87\left[(l, b)=\left(348^{\circ} .69,-0.77\right)\right]$ and WR $89\left[(l, b)=\left(348^{\circ} 72,-0.78\right)\right]$ are members of this cluster (van der Hucht 2001). Another WR star present in this area is WR $91\left[(l, b)=\left(348^{\circ} .76,-1^{\circ} .07\right)\right]$. This star does not belong to HM 1 and is seen projected onto RCW 122. For the sake of clarity, the main constituents and their relative location are shown in Fig. 1 superimposed on a Midcourse Space Experiment (MSX) Band A image of the region of interest.

In the radio band, RCW 122 appears as a small diameter source, 3 '. $2 \times 2$ '.8 at $4.85 \mathrm{GHz}$ (Wright et al. 1994), which is detectable from $0.408 \mathrm{MHz}$ (Shaver \& Goss 1970a) to $300 \mathrm{GHz}$ (Cheung et al. 1980; and Arnold et al. 1978). At most of the frequencies the source is observed projected onto a lowlying plateau of continuum emission that extends northwards (in equatorial coordinates) of RCW 122. Based on 0.408 and $5 \mathrm{GHz}$ data, Shaver \& Goss (1970b) find that this source is thermal and optically thick at the lower frequency. From the same set of measurements, they derived an emission measure (EM) of $\mathrm{EM}=9.3 \times 10^{5} \mathrm{pc} \mathrm{cm}^{-6}$. Adopting a distance of $2 \mathrm{kpc}$, Shaver \& Goss (1970b) derived an electron density $\left(n_{\mathrm{e}}\right)$ of $n_{\mathrm{e}} \sim 760 \mathrm{~cm}^{-3}$, a mass of ionized hydrogen of $M_{\mathrm{HII}} \sim 38 M_{\odot}$ and an excitation parameter $(U)$ of $U \simeq 67 \mathrm{pc} \mathrm{cm}^{-2}$.

Radio continuum observations of RCW 121 were carried out at 2.7 (Beard et al. 1969) and 4.85 GHz (Wright et al. 1994). 


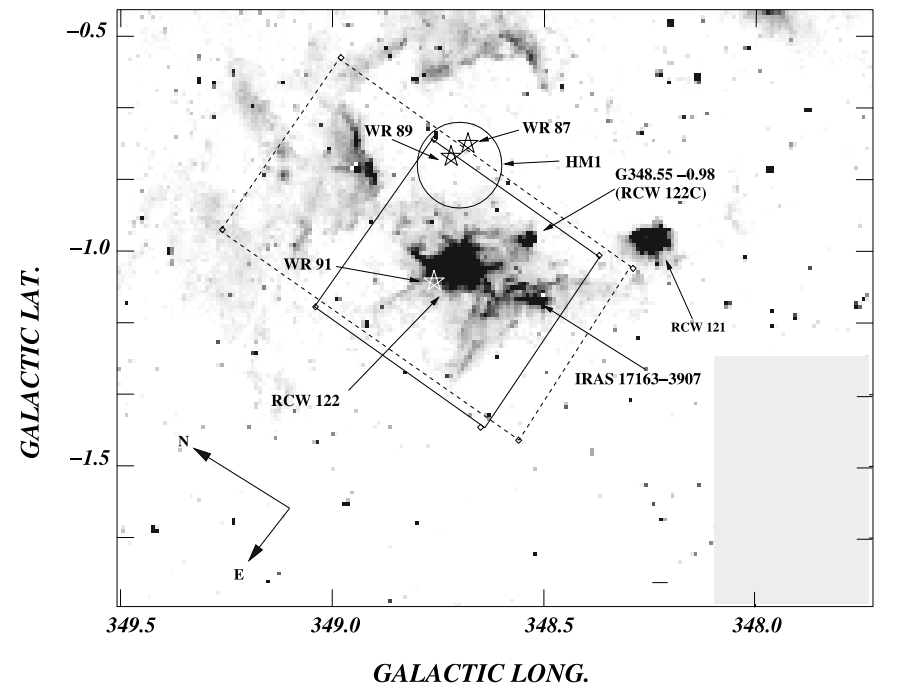

Fig. 1. Grey scale MSX A band image $(8.1 \mu \mathrm{m})$ of the region where RCW 121 and RCW 122 are located. A dark grey tonality indicate regions of high emissivity. The diameter of the circle corresponds to the optical diameter of the cluster HM 1. The position of the Wolf-Rayet stars is marked by a star symbol. The area observed by Gillespie et al. (1979) is delimited by the large dash rectangle. The small rectangle shows the region studied by Ghosh et al. (1989) in the far-IR. The NANTEN ${ }^{12} \mathrm{CO}$ observations cover the entire region, except for the grey rectangle shown in the lower right corner, depicted in this figure. The orientation of the equatorial coordinate system is given by the thick arrows labelled E (East) and N (North).

The angular diameter of RCW 121 at $4.85 \mathrm{GHz}$ is $2.7 \times 2.3$ (Wright et al. 1994).

At infrared wavelengths, RCW 122 was first observed by Emerson et al. (1973). They carried out observations with a broad bandwidth (half-power points at 40 and $350 \mu \mathrm{m}$ ) with an instrument having an angular resolution of 3.5. Adopting a distance of $5 \mathrm{kpc}$, they derived an overall luminosity of $17.3 \times 10^{5} L_{\odot}$. High-resolution (HPBW $\sim 1^{\prime}$ ) far-infrared observations of the region were carried out by McBreen et al. (1985) [40-250 $\mu \mathrm{m}$ ] and Ghosh et al. (1989) [120-300 $\mu \mathrm{m}$ ]. McBreen et al. (1985) resolved RCW 122 in two sources labelled A and B, respectively. These sources, RCW $122 \mathrm{~A}$ and RCW 122 B, from here onwards will be referred to as RCW 122. The former coincides with the peak of radio continuum emission, while RCW $122 \mathrm{~B}$, located 2'.6 southwest of RCW $122 \mathrm{~A}$, is seen projected onto an elongation of the main radio continuum source. Another source of this complex, RCW $122 \mathrm{C}$ ( $\equiv \mathrm{G} 348.55-0.98$ ), is located $\sim 10.5$ northwest of RCW $122 \mathrm{~A}$. Zhang et al. (1995) have carried out high-spatial resolution observations of RCW $122 \mathrm{C}$ at $50 \mu \mathrm{m}$ and $100 \mu \mathrm{m}$, resolving this source into two separate components spaced one from the other by $\sim 70^{\prime \prime}$. Very high resolution $\left(\mathrm{HPBW}=2^{\prime \prime} .8 \times 1^{\prime \prime}\right.$. 3 ) continuum observations of RCW $122 \mathrm{C}$ carried out by Gaume \& Mutel (1987) at $15 \mathrm{GHz}$, revealed the presence of one ultracompact and two extended HII regions. The extended regions coincide with the far-infrared sources detected by Zhang et al. (1995).

Recently, J, H, and K photometry of RCW 121 (RomanLopes \& Abraham 2006) unveiled the presence of a young cluster of massive stars embedded in an HII region coincident with RCW 121. Based on the size of the Br $\gamma$ emitting region and the detected 4.85 GHz continuum flux, Roman-Lopes \& Abraham (2006) derived an emission measure of $1.5 \times 10^{6} \mathrm{pc} \mathrm{cm}^{-6}$ and an electron density of $2.6 \times 10^{3} \mathrm{~cm}^{-3}$.
Radio recombination line (RRL) observations of RCW 122 and RCW 121 (Wilson et al. 1970; McGee \& Newton 1981; Shaver et al. 1983; Caswell \& Haynes 1987; Quireza et al. 2006a) show emission at a radial velocity of about $-12 /-13 \mathrm{~km} \mathrm{~s}^{-1}$ (for RCW 122) and $\sim 18 \mathrm{~km} \mathrm{~s}^{-1}$ (for RCW 121). Based on RRL data, the electron temperature, $T_{\mathrm{e}}$, of the ionized gas ranges between $6000 \mathrm{~K}$ (Shaver et al. 1983) and $7150 \mathrm{~K}$ (Quireza et al. 2006b) (for RCW 122) and between $5000 \mathrm{~K}$ (Caswell \& Haynes 1987) and 6610 K (Quireza et al. 2006b) (for RCW 121). All radial velocities refer to the Local Standard of Rest (LSR).

A wealth of molecular line emission arising from nonmasing (Juvela 1996; Gardner \& Whiteoak 1978; Whiteoak \& Gardner 1978; Batchelor et al. 1981; Whiteoak \& Gardner 1974; Martin et al. 1983; Brand et al. 1984; Whiteoak et al. 1982; and Sollins \& Megeath 2004) and masing (Kaufmann et al. 1976; Batchelor et al. 1980; Caswell 1997, 2003; Caswell et al. 1995; and Walsh et al. 1998) transitions were observed towards the peak of the continuum emission $\left[(l, b)=\left(348^{\circ} .7,-1^{\circ} .0\right)\right]$ of RCW 122 , using a large variety of single dish instruments. The radial velocity of the non-masing transitions fall in the range -16 to $-12.4 \mathrm{~km} \mathrm{~s}^{-1}$. Maser emission was also observed (Caswell et al. 1983; Gaume \& Mutel 1987; Caswell 1997, 2003; and Caswell et al. 1995) toward the ultracompact HII region of RCW $122 \mathrm{C}$.

CS $(J=2 \rightarrow 1)$ line emission (Bronfman et al. 1996) was observed toward IRAS17158-3901, a source likely to be associated with RCW 122 C and IRAS17149-3916, identified as the infrared counterpart of RCW 121 . The peak radial velocity of the CS line is $-13.4 \mathrm{~km} \mathrm{~s}^{-1}$ [IRAS17158-3901] and $-16.0 \mathrm{~km} \mathrm{~s}^{-1}$ [IRAS17149-3916], respectively.

Gillespie et al. (1979) surveyed at $\mathrm{CO}(J=1 \rightarrow 0)$ $(\mathrm{HPBW}=3.2)$ an area of 0.5 square degrees towards RCW 122. This area is shown in Fig. 1 as a dash rectangle. They found a peak of $\mathrm{CO}$ emission almost coincident with the radio continuum peak of RCW 122 as observed by Goss \& Shaver (1970). The radial velocity of this gas covers the range from -16.5 to $-9 \mathrm{~km} \mathrm{~s}^{-1}$.

The distance to RCW 121 and RCW 122 is a matter of some debate. Two different approaches have been used to determine their distances. On the one hand, based on the radial velocity of recombination line and/or molecular line observation, two kinematical distances are likely, namely a near-kinematical distance of $\sim 2 \mathrm{kpc}$ and a far-kinematical distance of $\sim 17 \mathrm{kpc}$ (Walsh et al. 1997, and references therein). Since both sources have optical counterparts, the near-kinematical distance is generally adopted. On the other hand, interferometer $21-\mathrm{cm}$ absorption measurements (Goss et al. 1970; Radhakrishnan et al. 1972) provide a distance estimate of $\sim 5 \mathrm{kpc}$.

A glance at Fig. 1 shows that the cluster HM 1 and its WolfRayet members, WR 87 and WR 89, are approximately located at the geometric centre of a large ring-like feature of IR emission. This feature has a low level of emission north and east (in galactic coordinates) of the cluster HM 1, but its emission becomes much stronger towards the south and southwest of this cluster. The star forming regions RCW 122 and RCW $122 \mathrm{C}$ are located in this area. We speculate that RCW 121, though a bit eccentric with respect to the geometric centre, may also belong to this large feature.

Expanding shells around WR stars have been observed at different wavelength ranges (Cappa 2006). It also has been speculated that in these shells mechanisms capable of triggering star formation may be at work (Whitworth et al. 1994). Bearing this in mind and given the peculiar distribution of the IR emission 
around HM 1 (see Fig. 1), it is worth further exploring a possible physical link among the IR arc-like feature, the WR stars and the open cluster HM 1. Are star forming regions RCW 121 and RCW 122 (A, B and C) triggered in an expanding shell blown away by the most massive members of HM 1? Are RCW 122 and $\mathrm{RCW} 122 \mathrm{C}$ and RCW 121 different star forming regions within the same molecular complex or star forming regions located in otherwise unrelated molecular clouds?

We carried out $115 \mathrm{GHz}{ }^{12} \mathrm{CO}(J=1 \rightarrow 0)$ observations covering a $\sim 2.1$ square degrees area aimed at answering these questions. The databases used in this study are briefly outlined in Sect. 2; results are reported in Sect. 3; and discussion in Sect. 4. Conclusions are presented in Sect. 5.

\section{Databases}

This research was carried out using:

i) Intermediate angular resolution, medium sensitivity, and high-velocity resolution ${ }^{12} \mathrm{CO}(J=1 \rightarrow 0)$ data obtained with the 4-m NANTEN millimeter-wave telescope of Nagoya University. At the time the authors carried out the observations, April 2001, this telescope was installed at Las Campanas Observatory, Chile. The halfpower beamwidth and the system temperature, including the atmospheric contribution towards the zenith, were 2'.6 $(\sim 3.8 \mathrm{pc}$ at $5 \mathrm{kpc})$ and $\sim 220 \mathrm{~K}(\mathrm{SSB})$ at $115 \mathrm{GHz}$. The data were gathered using the position switching mode. Observations of points devoid of $\mathrm{CO}$ emission were interspersed among the program positions. The coordinates of these points were retrieved from a database that was kindly made available to us by the NANTEN staff. The spectrometer used was an acusto-optical with 2048 channels providing a velocity resolution of $\sim 0.055 \mathrm{~km} \mathrm{~s}^{-1}$. For intensity calibrations, a room-temperature chopper wheel was employed (Penzias \& Burrus 1973). An absolute intensity calibration (Ulich \& Hass 1976; Kutner \& Ulich 1981) was achieved by observing Orion KL (RA $(1950.0)=5^{\mathrm{h}} 32^{\mathrm{m}} 47^{\mathrm{s}} .0, \quad$ Dec $\left.(1950.0)=-5^{\circ} 24^{\prime} 21^{\prime \prime}\right)$, and $\rho$ Oph East $\left(\right.$ RA $(1950.0)=16^{\mathrm{h}} 29^{\mathrm{m}} 20^{\mathrm{s}} .9$, Dec $(1950.0)=-$ $\left.24^{\circ} 22^{\prime} 13^{\prime \prime}\right)$. The absolute radiation temperature, $T_{R}^{*}$, of Orion KL and $\rho$ Oph East, as observed by the NANTEN radiotelescope were assumed to be $65 \mathrm{~K}$ and $15 \mathrm{~K}$, respectively (Moriguchi et al. 2001). The CO observations covered a region $(\Delta l \times \Delta b)$ of $101.5 \times 79$ !. centred at $(l, b)=\left(348^{\circ} .625,-1.115\right)$. An inner area of $31.2 \times 32 ! 4$ centred at $(l, b)=(348.76,-1.06)$ was sampled one beam apart, while the remaining area was sampled every 5:4 (two beamwidth). The integration time per point was 16 s resulting in a typical rms noise of $\sim 0.3 \mathrm{~K}$. Compared to the Gillespie et al. (1979) data, our database provides a slight improvement in resolution and an increase in sensitivity (at a velocity resolution similar to the one used by Gillespie et al. ) of a factor of 80 .

ii) New flux density determinations derived from radio continuum surveys carried out at $2.417 \mathrm{GHz}^{1}$ (Duncan et al. 1995), $5.0 \mathrm{GHz}^{2}$ (Haynes et al. 1978), and 8.75 and $14.35 \mathrm{GHz}^{3}$ (Langston et al. 2000), and flux density determinations at other frequencies available in the literature.

\footnotetext{
1 http://www.atnf.csiro.au/research/surveys/2.4Gh_Southern/

2 http://wwW/mpifr-bonn.mpg.de/survey.html

3 http://www.gb.nrao.edu/ glangsto/GPA/
}

iii) Infrared data retrieved from the Midcourse Space Experiment $(\mathrm{MSX})^{4}$ (Price et al. 2001) and the Improved Reprocessing of the IRAS Survey (IRIS) ${ }^{5}$ (Miville-Deschênes \& Lagache 2005).

\section{Observational results}

\subsection{Large scale CO distribution}

Figure 2 shows a series of representative mean emission CO profiles obtained at different positions around RCW 121 and RCW 122. Carbon monoxide emission is observed along three main velocity ranges: a) -38 to $-24 \mathrm{~km} \mathrm{~s}^{-1}$; b) -23 to $-8 \mathrm{~km} \mathrm{~s}^{-1}$; and c) -8 to $-2 \mathrm{~km} \mathrm{~s}^{-1}$.

Figure 2 shows that the strongest $\mathrm{CO}$ emission mostly falls in the velocity range from -20 to $-10 \mathrm{~km} \mathrm{~s}^{-1}$. This component is noticeable towards the lower half of Fig. 2 and achieves its maximum intensity towards RCW $122\left(T_{\mathrm{R}}^{*} \sim 21 \mathrm{~K}\right)$ and RCW 121 $\left(T_{\mathrm{R}}^{*} \sim 16 \mathrm{~K}\right)$. On the other hand, close to the galactic plane $(|b| \leq 0.85)$ the large scale $\mathrm{CO}$ emission (see Fig. 2 upper three panels) is much weaker $\left(T_{\mathrm{R}}^{*} \leq 3 \mathrm{~K}\right)$.

From Fig. 3 it is readily noticeable that the $\mathrm{CO}$ emission distribution is quite dissimilar among the three velocity ranges defined above. In the first one, -38 to $-24 \mathrm{~km} \mathrm{~s}^{-1}$, the $\mathrm{CO}$ emission is quite patchy and there is only weak emission $\left(T_{\mathrm{R}}^{*} \leq 0.8 \mathrm{~K}\right)$ towards both RCW 121 and RCW 122, while in the second one $\left(-23\right.$ to $\left.-8 \mathrm{~km} \mathrm{~s}^{-1}\right)$ the overall molecular emission distribution is dominated by a large structure having a low level of extended molecular emission and three strong $\mathrm{CO}$ concentrations. In order of decreasing $\mathrm{CO}$ peak intensity these concentrations are spatially correlated with RCW 122, RCW 121, and $\mathrm{RCW} 122 \mathrm{C}$. The large structure of molecular emission appears confined to galactic longitudes $347^{\circ} .8 \leq l \leq 348^{\circ}$. 9 and galactic latitudes $-1.7 \leq b \leq-0.75$. Along the third velocity range, -8 to $-2 \mathrm{~km} \mathrm{~s}^{-1}$, the $\mathrm{CO}$ emission is much weaker and mostly concentrated around $b \sim-1.0$. The strong molecular emission observed in this velocity range towards RCW $122 \mathrm{C}\left[(l, b)=\left(348^{\circ} .6,-1^{\circ} .0\right)\right]$ is not a new feature, but the positive velocity tail of the molecular emission observed towards this source at lower velocities.

Bearing in mind that RCW 121, RCW 122 (A, B, and C) are known young star-forming regions, and based on the fact that previous single-pointing molecular line observations of these sources show that molecular gas having a radial velocity of $\sim-15 \mathrm{~km} \mathrm{~s}^{-1}$ is likely to be associated with them, it is our understanding that most of the molecular gas observed in the velocity range from -23 to $-8 \mathrm{~km} \mathrm{~s}^{-1}$ (Fig. 3, upper right panel) may be physically related to these regions. In order to follow in more detail the spatial distribution of this gas, in Fig. 4 a mosaic of eight images is shown. These images cover the velocity range from -21 to $-5 \mathrm{~km} \mathrm{~s}^{-1}$, and every image represents a mean of the $\mathrm{CO}$ emission over a velocity interval of $\sim 2 \mathrm{~km} \mathrm{~s}^{-1}$. The central velocity of each image is indicated in the inner lower right corner. These narrow velocity channels show that the molecular gas distribution changes considerably along this velocity range. At $-20 \mathrm{~km} \mathrm{~s}^{-1}$, the $\mathrm{CO}$ emission shows quite a few molecular concentrations of similar strength spread over the observed region. At more positive velocities (Figs. 4b-d), most of the $\mathrm{CO}$ emission is concentrated on a large semi-annular feature observed at $l \leq 348.7$ and $b \leq-0.8$. This feature depicts a lot of small scale structures. The $\mathrm{CO}$ concentrations with the strongest emission (labelled A and B in Fig. 4c) have a good

\footnotetext{
4 http://irsa.ipac.caltech.edu/Missions/msx.html

5 http://www/ias.u-psud.fr/iris/
} 


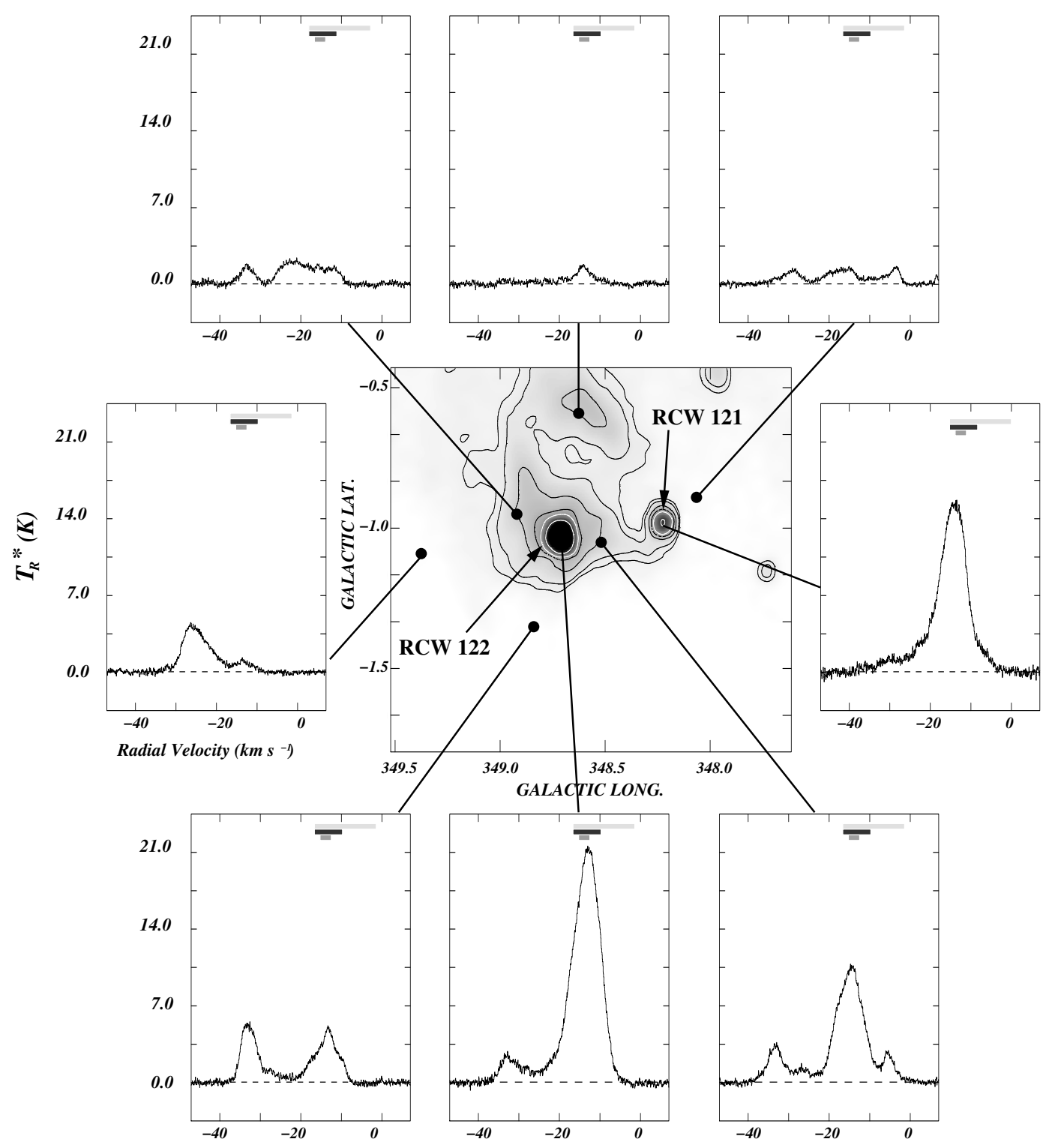

Fig. 2. Mean CO emission profiles toward eight regions (outer panels) around RCW 121 and RCW 122. The CO profiles are averaged over a square area $10^{\prime}$ in size. These squares are centred on the black dots drawn on the $5.0 \mathrm{GHz}$ (Haynes et al. 1978) continuum image (central panel, solid contours) of the region. The position of RCW 121 and RCW 122 are marked. The location of RCW $122 \mathrm{C}$ is almost coincident with the black dot corresponding to the lower right panel. Three grey bars at the top inner right corner of each panel represent the velocity range of recombination lines (dark grey), millimeter emission (intermediate grey), and maser emission (light grey) observed in the region by other researchers. The continuum image covers the same area depicted in Fig. 1 . The profile units are $T_{\mathrm{R}}^{*}$ in $\mathrm{K}$ (ordinate) and $V_{\mathrm{LSR}}$ in $\mathrm{km} \mathrm{s}^{-1}$ (abscissae).

morphological correspondence with RCW 121 (Feature B) and RCW 122 (Feature A). These features achieve their highest intensity at $-14 \mathrm{~km} \mathrm{~s}^{-1}$ (Fig. 4d) and fade away as we move towards more positive velocities. Another $\mathrm{CO}$ feature having a remarkable positional coincidence with an infrared feature is the one labelled C (Fig. 4e). This structure begins to be noticeable at $-14 \mathrm{~km} \mathrm{~s}^{-1}$ and can be traced all the way till $-6 \mathrm{~km} \mathrm{~s}^{-1}$. Feature $\mathrm{C}$ is seen projected onto RCW $122 \mathrm{C}$.

Figure 5 shows a striking correlation among the infrared counterparts of RCW 121, RCW 122 (A, B and C), and the $\mathrm{CO}$ distribution, in the sense that each major IR feature has its own CO counterpart. A southern extension of RCW 122 at $8.1 \mu \mathrm{m}$ is closely delineated by a CO feature that potrudes from the main body of CO emission observed towards RCW 122.

The $\mathrm{CO}$ features mentioned above are projected onto a much more extended $\mathrm{CO}$ structure that has a low level of emission.
From here onwards, this structure will be referred to as the plateau. Outside the region delimited by the plateau, there are four regions showing lower levels of $\mathrm{CO}$ emission $\left(T_{\mathrm{R}}^{*} \leq 9 \mathrm{~K}\right)$ compared to those observed towards the region engulfed by the plateau. The coordinates where these regions achieve their maximum CO intensity are $\left(349^{\circ} .23,-1^{\circ} .04\right),\left(349^{\circ} .10,-1^{\circ} 26\right)$, $\left(348^{\circ} .94,-0.68\right)$ and $\left(348^{\circ} .68,-0.53\right)$, respectively. Mean CO profiles obtained at each of these maxima show an emission profile that is different with respect to those observed towards the molecular cloud engulfing RCW 121, RCW 122, and RCW $122 \mathrm{C}$. The main differences are the shape, the number of emission features present in each spectrum, and the mean weighted (by temperature) velocity of these features. Figure 6 shows mean $\mathrm{CO}$ profile of the strongest regions.

This dissimilarity in appearance may indicate that the emission features seen in these spectra arise from regions physically 

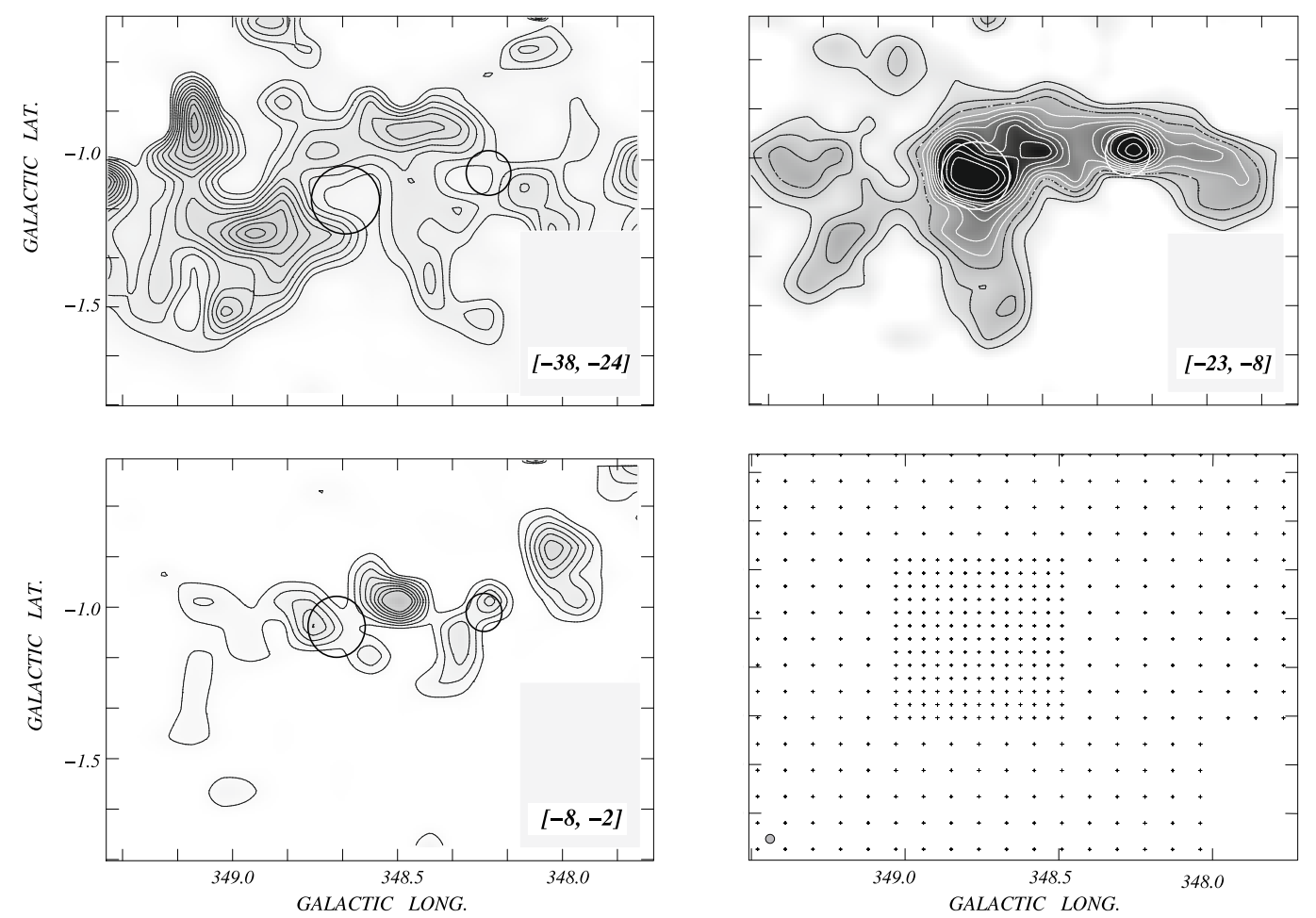

Fig. 3. Spatial distribution of $\mathrm{CO}$ emission in three velocity ranges. The velocity range is given in the inner lower right corner of each image. The small circumference represents the aproximate $5 \mathrm{GHz}$ location of RCW 121, while the large one represents the approximate location of RCW 122. The light grey boxes at the bottom right corner of every image represents areas not covered by the observations (see lower right panel). The grey scale minimum and maximum are 0.7 and $12.6 \mathrm{~K}$, respectively. The line temperature is an average over the velocity range indicated in the lower right corner of each panel. In the velocity range -38 to $-24 \mathrm{~km} \mathrm{~s}^{-1}$, the contour spacing is $0.3 \mathrm{~K}$ and the minimum and maximum temperatures are $0.85 \mathrm{~K}(\sim 14 \sigma)$ and $4.5 \mathrm{~K}$, respectively. In the range -23 to $-8 \mathrm{~km} \mathrm{~s}^{-1}$, the contour spacing is $0.7 \mathrm{~K}$ between the minimum temperature contour of $1.4 \mathrm{~K}(\sim 14 \sigma)$ till $7 \mathrm{~K}$ and $1.4 \mathrm{~K}$ from there onwards. In the range -8 to $-2 \mathrm{~km} \mathrm{~s}^{-1}$ the contour spacing is $0.35 \mathrm{~K}$, and the minimum and maximum contour temperature are $0.35 \mathrm{~K}(\sim 5 \sigma)$ and $2.8 \mathrm{~K}$, respectively. In the lower right panel the observed positions are marked by dots. The small circle in the lower left corner of this panel depicts the angular resolution of the NANTEN telescope.

unrelated to the plateau. Adopting the $14 \sigma$ level as its outer border, the plateau maximum angular extent in galactic longitude (at $b=-0.92$ ) is $\sim 10^{\circ}$, while in galactic latitude its dimensions vary between a maximum of $\sim 0.6$ (at $l \sim 348^{\circ} .7$ ) and a minimum of $\sim 0.23$ (at $l \sim 348^{\circ}$. 2). The deconvolved cloud size (to the $6.3 \mathrm{~K}$ level) of the CO structure related to RCW 121 is $\sim 0.3 \times 0.16$. For RCW $122 \mathrm{C}$ and RCW 122 (to the $7.0 \mathrm{~K}$ level), the CO features have an angular size of $\sim 0.27 \times 0^{\circ} .13$ and $\sim 0.34 \times 0.34$, respectively.

The mass of the molecular gas traced by $\mathrm{CO}$ can be estimated using the empirical relationship between the molecular hydrogen column density, $N\left(\mathrm{H}_{2}\right)$, and the integrated molecular emission, $W(\mathrm{CO})\left(\equiv \int T_{\mathrm{R}}^{*} \mathrm{~d} v\right)$, over a given velocity range. The conversion between $W(\mathrm{CO})$ and $N\left(\mathrm{H}_{2}\right)$ is given by the equation

$N\left(\mathrm{H}_{2}\right)=(1.9 \pm 0.3) \times 10^{20} W(\mathrm{CO}),\left(\mathrm{cm}^{-2}\right)$

which we obtained from $\gamma$-ray studies of molecular clouds in the Cepheus Flare (Digel et al. 1996). The mass of the molecular concentration, $M\left(\mathrm{H}_{2}\right)$, was calculated with the equation

$M_{\mathrm{tot}}=\mu m_{\mathrm{H}} \sum \Omega N\left(\mathrm{H}_{2}\right) d^{2}\left(M_{\odot}\right)$

where $\mu$ is the mean molecular weight, assumed to be equal to 2.76 after allowance of a relative helium abundance of $25 \%$ by mass; $m_{\mathrm{H}}$ is the hydrogen atom mass; $\Omega$ is the solid angle subtended by the CO feature; and $d$ is its distance, expressed in kpc. The distance of $5 \mathrm{kpc}$ derived from HI absorption experiments by Radhakrishnan et al. (1972) will be adopted for these sources. We use a conservative lower distance uncertainty of $\pm 0.5 \mathrm{kpc}$. This distance uncertainty, plus the one quoted for the coefficient of Eq. (1), implies a total mass uncertainty of the order of $\sim 26 \%$. The summation was performed over those pixels having a $T_{\mathrm{R}}^{*} \geq 7.0 \mathrm{~K}$ for RCW 122 and RCW $122 \mathrm{C}$ ), and $T_{\mathrm{R}}^{*} \geq 6.3 \mathrm{~K}$ for RCW 121 . The mean volume density of molecular hydrogen of each structure is derived from the ratio of the total molecular mass and the volume of an ellipsoid whose major $\left(d_{\mathrm{M}}\right)$ and minor $\left(d_{\mathrm{m}}\right)$ axis are given in Table 1. The latter are derived from the $50 \sigma$ contour level. It is worth mentioning that using the CO map of Gillespie et al. (1979), McBreen et al. (1985) estimate a total molecular mass of $\sim 3.4 \times 10^{5} \mathrm{M}_{\odot}$. This estimate is a factor of 3.5 lower than the value quoted in Table 1. Very likely this difference has its origin in the quite different noise level of the involved databases.

Due to the large angular dimensions of the $\mathrm{CO}$ structure seen projected onto RCW 122 (Feature A) and the better spatial sampling of the observed points in this area, 21 independent $\mathrm{CO}$ profiles are observed towards the region enclosed by the $11.9 \mathrm{~K}$ contour line in Fig. 5. Having all these spectra a high signal-to-noise $(S / N)$ ratio $(S / N \geq 40)$, they are well suited to study in some detail possible variations of the $\mathrm{CO}$ emission profiles across the region. To this end, we used the Astronomical Image Processing System (AIPS) package to calculate the first four moments (integrated area, temperature weighted mean radial velocity, width and lopsidedness) of the $\mathrm{CO}$ profiles, in the velocity range from -20 to $-8.5 \mathrm{~km} \mathrm{~s}^{-1}$. Within the region defined by the $11.9 \mathrm{~K}$ contour line of Fig. 5 the mean weighted (by 

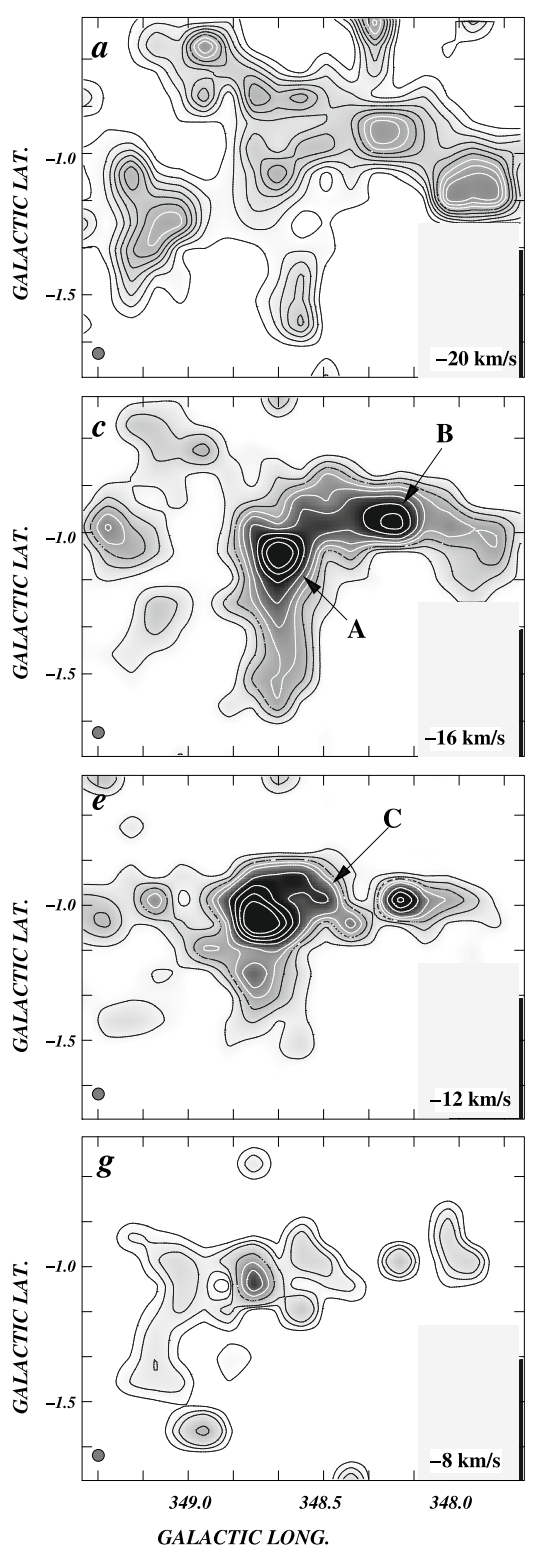
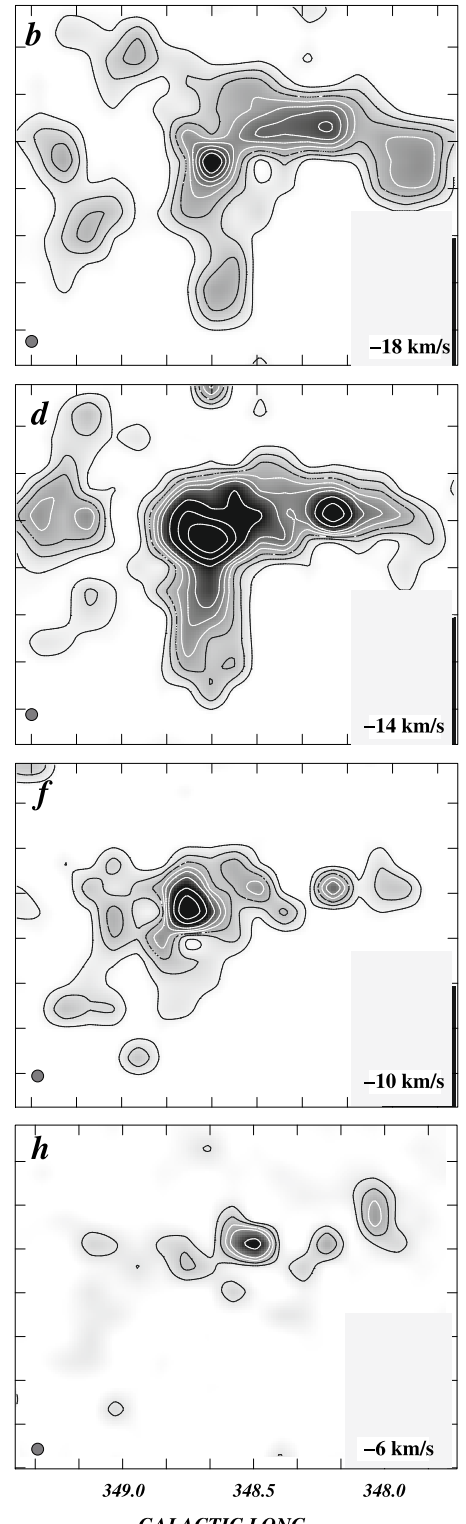

Fig. 4. Mean CO temperature line emission for selected velocity ranges. The velocity width of each map is $2 \mathrm{~km} \mathrm{~s}^{-1}$ and the displayed temperatures is a mean over the map velocity width. The central velocities are given at the lower right corner of each map. The beam size of the observations is shown as a shaded circle in the lower left corner of each map. The grey boxes at the bottom right corners represents sky regions that were not covered by our observations. The temperature contours are: for panel a) $0.7 \mathrm{~K}(\sim 13 \sigma)$ to $6.3 \mathrm{~K}$ with a contour spacing of $0.7 \mathrm{~K}$ and $7.7 \mathrm{~K}$ to $12 \mathrm{~K}$ with a contour spacing of $1.4 \mathrm{~K}$; for panel b) $0.7 \mathrm{~K}(\sim 13 \sigma)$ to $6.3 \mathrm{~K}$ with a contour spacing of $0.7 \mathrm{~K}$; for panel c) $1.4 \mathrm{~K}$ $(\sim 26 \sigma)$ to $11.2 \mathrm{~K}$ with a contour spacing of $1.4 \mathrm{~K}$; for panel d) $1.4 \mathrm{~K}(\sim 13 \sigma)$ to $6.4 \mathrm{~K}$ with a contour spacing of $1.4 \mathrm{~K}$ and $9.1 \mathrm{~K}$ to $17.5 \mathrm{~K}$ with a contour spacing of $2.8 \mathrm{~K}$; for panel e) $1.4 \mathrm{~K}(\sim 26 \sigma)$ to $8.4 \mathrm{~K}$ with a contour spacing of $1.4 \mathrm{~K}$ and $12 \mathrm{~K}$ to $19 \mathrm{~K}$ with a contour spacing of $3.5 \mathrm{~K}$; for panel f) $1.4 \mathrm{~K}(26 \sim \sigma)$ to $7 \mathrm{~K}$ with a contour spacing of $1.4 \mathrm{~K}$ and $10.5 \mathrm{~K}$ to $19 \mathrm{~K}$ with a contour spacing of $2.8 \mathrm{~K}$; for panel $\mathrm{g}) 1.4 \mathrm{~K}(\sim 26 \sigma)$ to $7 \mathrm{~K}$ with a contour spacing of $1.4 \mathrm{~K}$ and $9.1 \mathrm{~K}$ to $17.5 \mathrm{~K}$ with a contour spacing of $4.2 \mathrm{~K}$; and for panel $\mathbf{h}) 0.7 \mathrm{~K}(\sim 13 \sigma)$ to $2.1 \mathrm{~K}$ with a contour spacing of $0.7 \mathrm{~K}$ and $4.2 \mathrm{~K}$ to $14.7 \mathrm{~K}$ with a contour spacing of $2.1 \mathrm{~K}$. temperature) radial velocity is $V_{\mathrm{w}}=-13.6 \pm 0.9 \mathrm{~km} \mathrm{~s}^{-1}$. The quoted uncertainty is not a formal error, but reflects the variations of the radial velocity across Feature A. These variations follow a clear trend in the sense that, at galactic longitudes larger than $l \sim 348.7, V_{\mathrm{w}} \simeq-13.0 \pm 0.4 \mathrm{~km} \mathrm{~s}^{-1}$, while at lower longitudes $V_{\mathrm{w}} \simeq-14.5 \pm 0.3 \mathrm{~km} \mathrm{~s}^{-1}$. The width of the CO profile is $6.2 \pm 0.3 \mathrm{~km} \mathrm{~s}^{-1}$ and shows some variations across the region. Though it varies between a minimum of $5.4 \mathrm{~km} \mathrm{~s}^{-1}$ and reaches a maximum of $6.8 \mathrm{~km} \mathrm{~s}^{-1}$, unlike the radial velocity there is no clear spatial pattern for such variations. The behaviour of the third central moment, called skewness, is quite illustrative of the complexity of the dynamics of the molecular gas in this region. By definition, any symmetric distribution will have a third central moment equal to zero. If the distribution were skewed in such a way that there is a tail of emission toward the negative velocities, the skewness will be negative. Otherwise it will be positive. Over a region $4.1 \times 4.2$ centred at $(l, b)=\left(348^{\circ} .78\right.$, $-1.03)$, the skewness is $-2.3 \pm 0.2 \mathrm{~km} \mathrm{~s}^{-1}$ and, over a region of $5.2 \times 4.4$ centred at $(l, b)=\left(348^{\circ} .64,-1^{\circ} .12\right)$, the skewness is $+1.6 \pm 0.2 \mathrm{~km} \mathrm{~s}^{-1}$.

Therefore, the rapidly changing appearance (with position) of the $\mathrm{CO}$ profiles, plus the observed changes in $V_{\mathrm{w}}$, profile width and profile skewness across the face of RCW 122, point to the complex dynamics of the molecular gas emission observed towards this star forming region. Unfortunately, our data lack the angular resolution and spatial sampling required to undertake a more thorough study.

The mean kinematical properties of the molecular gas related to RCW $122 \mathrm{C}$ are derived from an analysis of six CO profiles observed within the $9.8 \mathrm{~K}$ contour line of Fig. 5. The mean weighted velocity is $V_{\mathrm{w}}=-14.9 \pm 0.5 \mathrm{~km} \mathrm{~s}^{-1}$ and the mean profile width is $6.9 \pm 0.3 \mathrm{~km} \mathrm{~s}^{-1}$. The individual profiles show shoulders and/or line asymmetries that are indicative of the presence of more than one component.

Owing to the coarse grid spacing of the $\mathrm{CO}$ data observed towards RCW $121(\Delta l \equiv \Delta b \equiv 5.4)$ the characteristic kinematical parameters of the molecular gas likely to be associated with this source were derived from only one CO profile observed close to the IR peak. From this spectrum a mean weighted (by temperature) velocity of $-16.3 \mathrm{~km} \mathrm{~s}^{-1}$ and a width of $\sim 4.6 \mathrm{~km} \mathrm{~s}^{-1}$ were derived.

In the overlay of the mean CO emission and the MSX A $(8.1 \mu \mathrm{m})$ image of the region shown in Fig. 5, there is no $\mathrm{CO}$ emission, above a $3-\sigma$ level, connecting the plateau with 


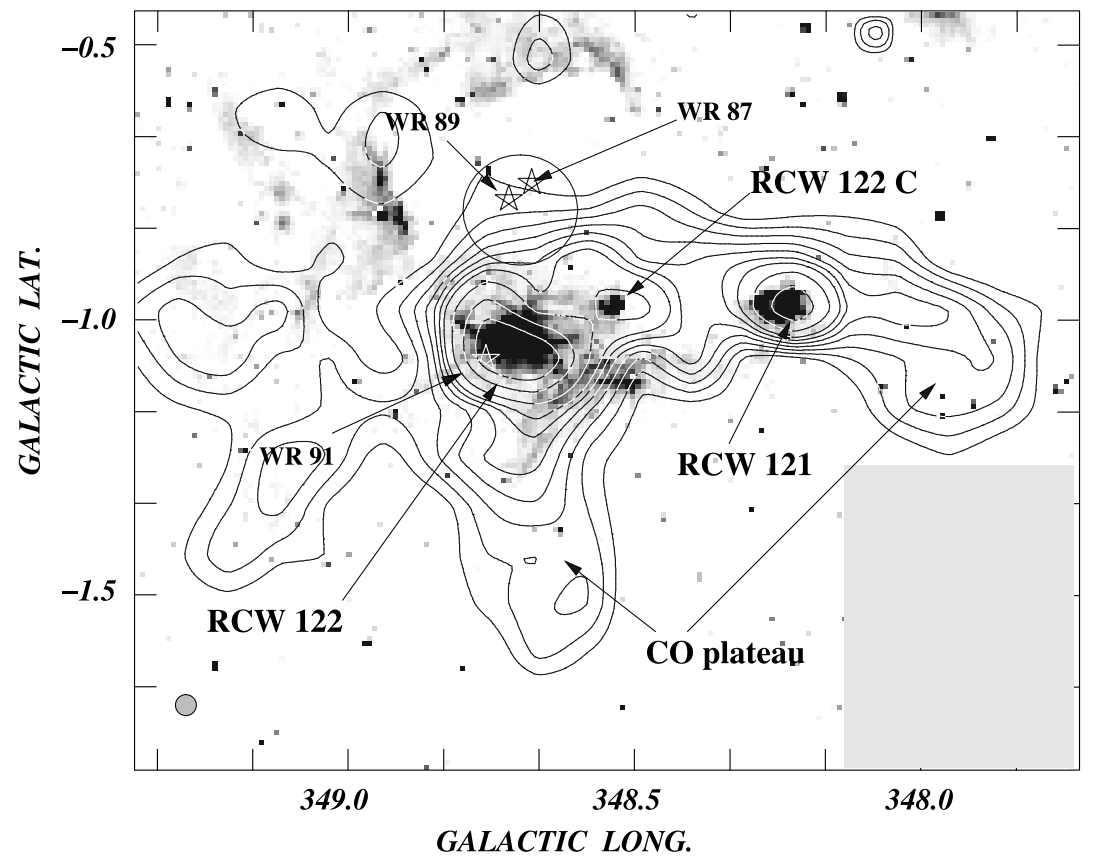

Fig. 5. Overlay of the mean $\mathrm{CO}$ temperature line emission (contours) along the velocity range -18 to $-5 \mathrm{~km} \mathrm{~s}^{-1}$ and MSX Band A $(8.1 \mu \mathrm{m})$ emission (grey scale). The temperature values are mean values over the entire velocity range. The lowest contour level is $1.4 \mathrm{~K}(\sim 14 \sigma)$, and the contour spacing is $0.7 \mathrm{~K}$ till $5.6 \mathrm{~K}$. From there onwards the contour spacing is $2.1 \mathrm{~K}$. The beam size of the $\mathrm{CO}$ observations is shown as a shaded circle in the lower left corner. the ring-like IR feature at whose geometric centre the open cluster HM 1 and the Wolf-Rayet stars WR 87 and WR 89 are seen projected. The lack of a "common" low level envelope of $\mathrm{CO}$ emission engulfing the stellar objects mentioned above and the plateau may be understood if the latter is unrelated to the former. This interpretation is in line with a distance determination of $2.9 \mathrm{kpc}$ (van der Hucht 2001) for WR 87 and WR 89 and HM 1.

The mean physical parameters (mass, volume density, linear size, and so forth) and a summary of the kinematical parameters of the major $\mathrm{CO}$ features are given in Table 1. The coordinates quoted in this table correspond to the maximum of the $\mathrm{CO}$ emission. The visual extinction listed in the eleventh row is derived from the relationship $N\left(\mathrm{H}_{2}\right) / A_{\mathrm{v}}=0.94 \times 10^{21} \mathrm{~cm}^{-2} \mathrm{mag}^{-1}$ (Bohlin et al. 1978).

\subsection{Radio continuum}

Making use of the digital radio continuum databases mentioned in Sect. 2, we obtained new flux density determinations at 2.417, 5.0, 8.35, and $14.35 \mathrm{GHz}$ for RCW 121 and RCW 122, respectively. The continuum data were processed using the NOD2 package (Haslam 1974). At a given frequency, the rms flux density noise level was determined from a source free region located in the neighbourhood of both sources. The rms flux density noise level is $0.02 \mathrm{Jy} /$ beam $(2.417 \mathrm{GHz})$; $0.03 \mathrm{Jy} /$ beam $(5.0 \mathrm{GHz})$; $0.12 \mathrm{Jy} /$ beam $(8.35 \mathrm{GHz})$; and $0.5 \mathrm{Jy} /$ beam $(14.35 \mathrm{GHz})$, respectively. The new flux density determinations, along with previous ones found in the literature, are summarised in Table 2 and the flux density spectrum of each source is shown in Fig. 7. We have placed all the measurements on the same absolute flux density scale given in Baars et al. (1977). The angular diameter of the continuum sources were derived from a two-dimensional Gaussian fit to the $5 \mathrm{GHz}$ database. Full width at half-maximum, corrected for beam broadening, of 3.2 $\times 2.5$ and 3.5 $\times 2$. 8 were obtained for RCW 121 and RCW 122, respectively. As can be seen from Fig. 7, RCW 122 has a spectrum characteristic of an HII region. At $408 \mathrm{MHz}$ this source appears to be optically thick, while at frequencies above $1.6 \mathrm{GHz}$ it exhibits a spectrum whose frequency behaviour is the one expected for an opticallythin plasma. The observed fluxes at $300 \mathrm{GHz}$ are well above the free-free flux density estimated by extrapolation of the lowfrequency data. This excess of infrared emission is attributed to thermal emission from heated dust grains (Cheung et al. 1980). In Fig. 7, the measurement at $2.7 \mathrm{GHz}$ (Beard et al. 1969) is likely to be an overestimate, for the authors quote this source as extended, while it is a point-like source at all other frequencies. Excluding this flux density as well as those measurements at $300 \mathrm{GHz}$, a weighted least square fit to the flux density measurements betweeen 1.6 and $85 \mathrm{GHz}$ gives a spectral index of $\alpha=-0.14 \pm 0.03\left(S_{v} \propto v^{\alpha}\right)$.

In order to determine physical parameters of the HII regions, we use the observed peak brightness temperature, $18.7 \pm 1 \mathrm{~K}$, of the radio continuum emission of RCW 122 at $5 \mathrm{GHz}$. After correcting for beam smearing - the ratio of the source solid angle to the main beam solid angle - this temperature is $\sim 29.4 \pm 1.6 \mathrm{~K}$. The observed brightness temperature $T_{\mathrm{b}}$ at frequency $v$ of an HII region having an electron temperature $T_{\mathrm{e}}$ is related to the plasma optical depth $\tau$ by

$$
T_{\mathrm{b}}(v)=T_{\mathrm{e}} \times\left(1 .-\mathrm{e}^{-\tau(v)}\right)
$$

where the optical depth at frequency $v$ is given by

$\tau=8.235 \times 10^{-2} T_{\mathrm{e}}^{-1.35} v^{-2.1} \mathrm{EM}$

where $T_{\mathrm{e}}$ is given in $\mathrm{K}$, the frequency $v$ is given in units of $\mathrm{GHz}$, and $\mathrm{EM}$ is the emission measure $\left(\mathrm{EM}=\int n_{\mathrm{e}}^{2} \mathrm{~d} l\right)$ given in units of $\mathrm{pc} \mathrm{cm}^{-6}$. Adopting an electron temperature of $T_{\mathrm{e}}=7150 \pm$ $90 \mathrm{~K}$, (Quireza et al. 2006a) from Eq. (3) an optical depth of $\tau=(4.1 \pm 0.3) \times 10^{-3}$ is derived, and from Eq. (4) the emission measure is $\mathrm{EM}=\left(2.3_{-0.1}^{+0.2}\right) \times 10^{5} \mathrm{pc} \mathrm{cm}^{-6}$.

For a pure hydrogen plasma, adopting an extent along the line of sight equal to the observed minor axis, $\sim 4.1 \mathrm{pc}$ at a distance of $5 \mathrm{kpc}$, and using the EM determined above, an electron density of $n_{\mathrm{e}}=240 \pm 10 \mathrm{~cm}^{-3}$ is derived. If this density were uniformely distributed on a spherical source whose equivalent angular diameter $\left(\Theta_{\mathrm{sph}}\right)$ is obtained from the observed half-power width of the Gaussian source, $\Theta_{g}=\sqrt{\Theta_{M} \times \Theta_{m}}$, 

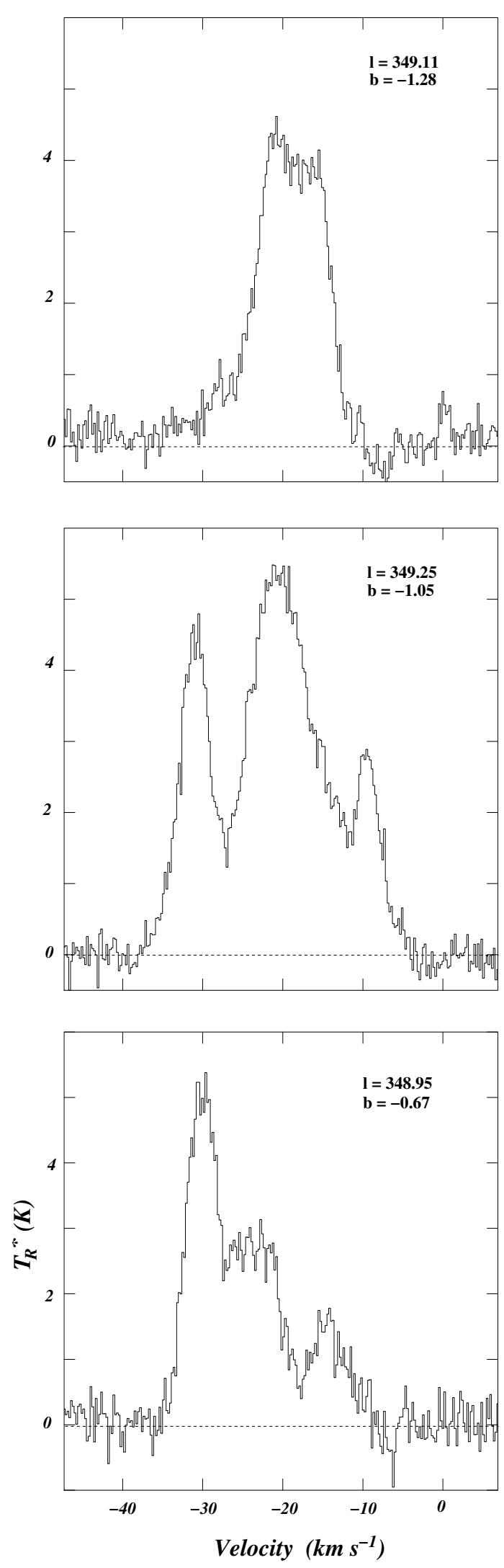

Fig. 6. Mean $\mathrm{CO}$ emission profiles toward three regions located outside the plateau (see text). These profiles were produced within AIPS using the task ISPEC. The $T_{\mathrm{R}}^{*}$ value given in the ordinate axis is a mean over a rectangular area, whose approximate centre is given in the upper right corner of each panel. The size of the rectangular areas are $19^{\prime} \times 7^{\prime}$ (upper panel); 12 '.5 $\times 13^{\prime}$ (middle panel); and 10' $\times 5$ '.4 (lower panel). The profile units are $T_{\mathrm{R}}^{*}$ in $\mathrm{K}$ (ordinates) and $V_{\mathrm{LSR}}$ in $\mathrm{km} \mathrm{s}^{-1}$ (abscissae).
Table 1. Mean physical parameters of the CO features.

\begin{tabular}{lrrrr}
\hline \hline Parameter & Feature A & Feature B & Feature C & Plateau \\
\hline Gal. Long. $\left(^{\circ}\right)$ & $348^{\circ} .70$ & 348.22 & 348.50 & - \\
Gal. Lat. $\left(^{\circ}\right)$ & -1.07 & -0.97 & -0.97 & - \\
$N\left(\mathrm{H}_{2}\right)\left(10^{22} \mathrm{~cm}^{-2}\right)$ & 1.8 & 1.0 & 0.7 & 1.2 \\
$\Omega\left(10^{-5} \mathrm{sr}\right)$ & 2.28 & 1.04 & 1.21 & 16.1 \\
$M_{\text {tot }}\left(10^{4} M_{\odot}\right)$ & 22.0 & 5.7 & 4.6 & 90.0 \\
major axis $\left(d_{\mathrm{M}}\right)(\mathrm{pc})$ & 30 & 25 & 24 & 96 \\
minor axis $\left(d_{\mathrm{m}}\right)(\mathrm{pc})$ & 21 & 14 & 11 & 21 to 52 \\
$n_{\mathrm{H} 2}\left(\mathrm{~cm}^{-3}\right)$ & 670 & 630 & 460 & 120 \\
$V_{\mathrm{w}}\left(\mathrm{km} \mathrm{s}^{-1}\right)$ & $-13.6 \pm 0.9$ & -16.3 & $-14.9 \pm 0.5$ & $-15 \pm 1.0$ \\
$\triangle V\left(\mathrm{~km} \mathrm{~s}^{-1}\right)$ & $6.2 \pm 0.3$ & 4.6 & $6.9 \pm 0.3$ & $3.0 \pm 0.7$ \\
$A_{\mathrm{v}}\left(\mathrm{mag}^{-1}\right)$ & $\sim 20$ & $\sim 11$ & $\sim 8$ & $\sim 13$ \\
Remarks & $\mathrm{RCW} 122$ & $\mathrm{RCW} 121$ & $\mathrm{RCW} 122 \mathrm{C}$ & - \\
\hline
\end{tabular}

which is defined as the geometric mean of the deconvolved major $\left(\Theta_{\mathrm{M}}\right)$ and minor $\left(\Theta_{\mathrm{m}}\right)$ angular dimensions. Recalling that $\Theta_{\text {sph }}=1.471 \times \Theta_{\mathrm{g}}($ Schraml \& Mezger 1969), a total mass of ionized gas of $M_{\mathrm{HII}}=920 \pm 40 M_{\odot}$ is derived. The turnover frequency of this source is $355_{-10}^{+20} \mathrm{MHz}$. For a plasma in which a fraction $x$ (by number) of the ions arise from singly-ionized helium, and $(1-x)$ from ionized hydrogen, the total mass of ionized gas must be increased by a factor $(1+3 x)$.

Alternatively, the electron density $\left(n_{\mathrm{e}}\right)$, the ionized mass $\left(M_{\mathrm{HII}}\right)$, and the central emission measured (EM) also can be obtained using simple geometric models for the HII regions (Schraml \& Mezger 1969). Using their spherical model (see Eqs. (3), (5)-(7), respectively, in their paper), we derived $n_{\mathrm{e}}=$ $252 \pm 6, M_{\mathrm{HII}}=980 \pm 25 M_{\odot}$ and $\mathrm{EM}=(4.3 \pm 0.2) \times 10^{5} \mathrm{pc} \mathrm{cm}^{-6}$. These figures are in good agreement with the values derived from the observed peak continuum brightness temperature.

The number of ionizing Lyman continuum photons $N_{\text {Lyc }}$ required to maintain the ionization of the source can be calculated from the continuum flux density measurements using the equation given by Simpson \& Rubin (1990)

$N_{\text {Lyc }}=5.25 \times 10^{48} T_{\mathrm{e}}^{-0.45} S_{v}(5 \mathrm{GHz}) d^{2}\left(\mathrm{~s}^{-1}\right)$.

Inserting in Eq. (5) the flux density given in Table 2, it is found that $N_{\text {Lyc }} \sim(9.3 \pm 0.3) \times 10^{49} \mathrm{~s}^{-1}$. This number is a lower limit to the total number of Lyman continuum photons produced by the stellar ionizing source, since a fraction of them are absorbed by dust present inside the HII region, showing up as the IRAS sources. Considering the relation between the number of Lyman continuum photons and spectral type given by Schaerer \& de Koter (1997), a minimum of $\sim 8$ O7 V stars would be needed to power RCW 122.

The radio continuum spectrum of RCW 121 is shown in the lower panel of Fig. 7. A weighted least-square fit gives a spectral index of $\alpha=-0.02 \pm 0.05$, which is interpreted as arising from an optically-thin thermal plasma. In this determination, the $2.7 \mathrm{GHz}$ flux density measurement was excluded from the fit.

The observed peak of the radio continuum emission of this source at $5 \mathrm{GHz}$ is $6.8 \pm 1 \mathrm{~K}$. After correcting for beam smearing this temperature increases to $\sim 10 \pm 1.5 \mathrm{~K}$. Adopting an electron temperature $T_{\mathrm{e}}=6610 \pm 100 \mathrm{~K}$ (Quireza et al. 2006b) and following the same procedure used before for RCW 122, we derived a continuum optical depth $\tau=\left(1.5_{-0.2}^{+0.3}\right) \times 10^{-3}$; an emission measure $\mathrm{EM}=\left(7.7_{-1.1}^{+1.2}\right) \times 10^{4} \mathrm{pc} \mathrm{cm}^{-6}$; a mean electron density of $n_{\mathrm{e}}=140 \pm 10 \mathrm{~cm}^{-3}$; a mass of ionized gas of $M_{\mathrm{HII}}=$ $390 \pm 30 M_{\odot}$; and a turnover frequency of $220 \pm 20 \mathrm{MHz}$. On the other hand, from the equations of Schraml \& Mezger (1969) we derived an emission measure $\mathrm{EM}=\left(1.7_{-1.7}^{+1.8}\right) \times 10^{5} \mathrm{pc} \mathrm{cm}^{-6}$; 


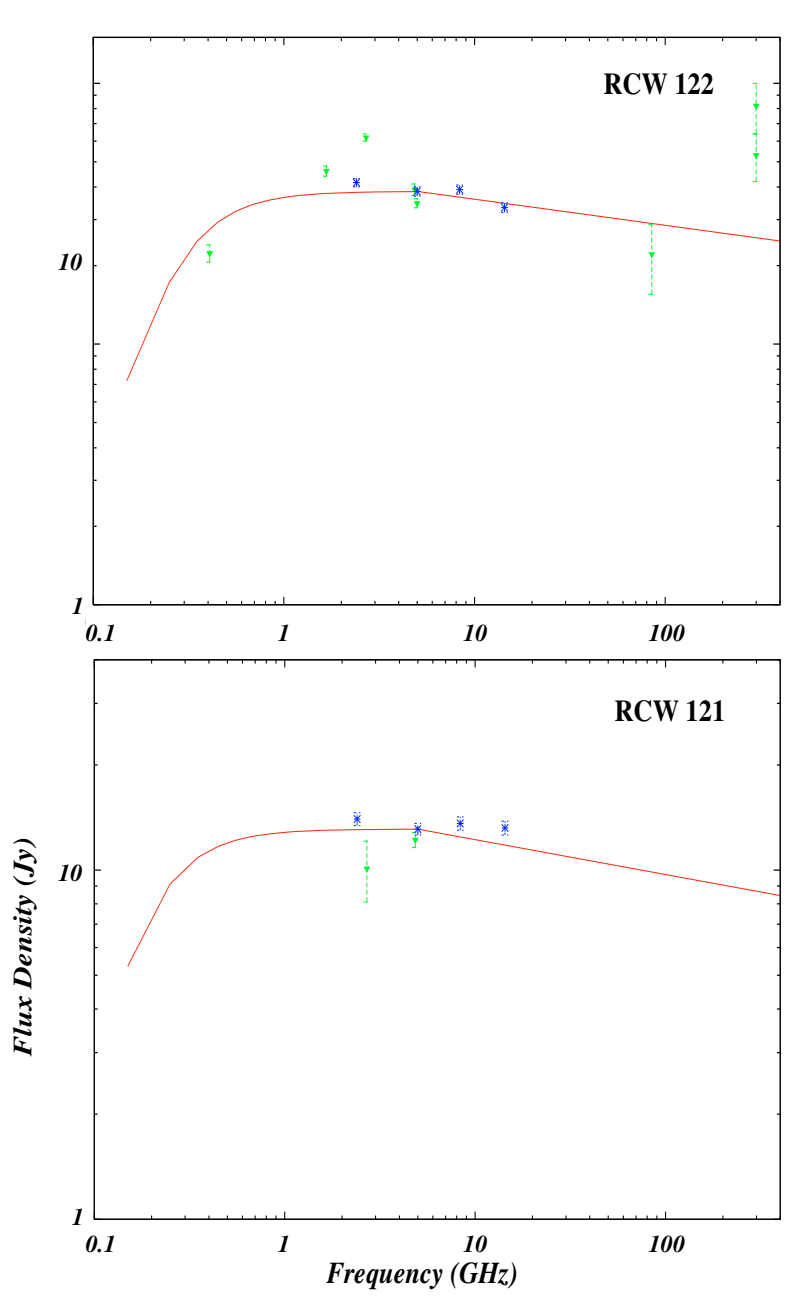

Fig. 7. Radio spectrum of RCW 122 (upper panel) and RCW 121 (lower panel). The asterisks identify those flux density determinations carried out in this paper, while filled inverted triangles identify flux density determinations available in the literature. The solid line represents the expected flux density for a source having a given emission measure and a given electron temperature. For RCW $122 \mathrm{EM}=2.3 \times 10^{5} \mathrm{pc} \mathrm{cm}^{-6}$ and $T_{\mathrm{e}}=7150 \mathrm{~K}$, while for RCW $121 \mathrm{EM}=7.7 \times 10^{4} \mathrm{pc} \mathrm{cm}^{-6}$ and $T_{\mathrm{e}}=$ $6610 \mathrm{~K}$. The observed spectrum of RCW 122 is consistent with this source being optically thick at $408 \mathrm{MHz}$, as claimed by Shaver \& Goss (1970b).

a mean electron density of $n_{\mathrm{e}}=170 \pm 5 \mathrm{~cm}^{-3}$; and a mass of ionized gas of $M_{\mathrm{HII}}=480 \pm 10 M_{\odot}$. Again, the electron density, the emission measure, and the total mass of ionized gas are in good agreement with those estimates derived from the observed peak continuum brightness temperature.

From the continuum data, the total number of ionizing photons needed to power this source is $N_{\text {Lyc }} \sim(3.3 \pm 0.1) \times 10^{49} \mathrm{~s}^{-1}$. In this case, at least $\sim 407 \mathrm{~V}$ stars would be needed to keep the present level of ionization.

\subsection{Mid- and far-infrared emission}

The IR emission distribution, at two different wavelengths, observed over the region covered by the $\mathrm{CO}$ observations is shown in Fig. 8. The MSX $8.13 \mu \mathrm{m}$ emission is shown on the left panel, while the IRAS $100 \mu \mathrm{m}$ emission is depicted on the right one. At both frequencies, strong emission is observed arising from the location of RCW 122, RCW 121, and RCW 122C (G348.55-0.98). The latter coincides in position with the IRAS
Table 2. Continuum flux density measurements.

\begin{tabular}{lrrl}
\hline \hline $\begin{array}{l}\text { Frequency } \\
(\mathrm{GHz})\end{array}$ & $\begin{array}{r}\text { RCW 121 } \\
(\mathrm{Jy})\end{array}$ & $\begin{array}{r}\text { RCW 122 } \\
(\mathrm{Jy})\end{array}$ & Remarks \\
\hline 0.408 & & $22.3 \pm 1.7$ & Shaver \& Goss (1970a) \\
1.67 & - & $46.1 \pm 2.1$ & Caswell \& Robinson (1974) \\
2.417 & $13.5 \pm 0.5$ & $41.6 \pm 1.5$ & This paper \\
2.7 & $10.1 \pm 2.0$ & $62.0 \pm 2.0$ & Beard et al. (1969) \\
4.85 & $12.2 \pm 0.6$ & $39.1 \pm 2.0$ & Wright et al. (1994) \\
5.0 & $13.1 \pm 0.5$ & $38.5 \pm 1.6$ & This paper \\
5.0 & - & $34.7 \pm 1.4$ & Goss \& Shaver (1970) \\
8.35 & $13.6 \pm 0.6$ & $39.1 \pm 1.6$ & This paper \\
14.35 & $13.2 \pm 0.6$ & $33.4 \pm 1.4$ & This paper \\
85.0 & - & $22.1 \pm 6.6$ & Brown \& Broderick (1973) \\
300.0 & - & $53.0 \pm 11.0$ & Cheung et al. \\
300.0 & - & $82.0 \pm 18.0$ & Arnold et al. (1978) \\
\hline
\end{tabular}
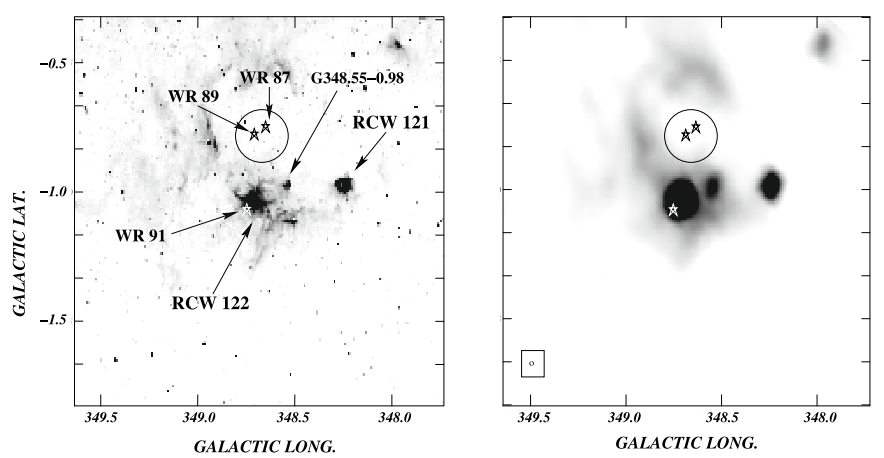

Fig. 8. Extended IR emission towards RCW 121 and RCW 122 at $8.13 \mu \mathrm{m}$ (MSX Band A, left panel) and $100 \mu \mathrm{m}$ (IRIS data, right panel). The position of the infrared counterparts of RCW 121 and RCW 122, and strong sources are indicated in the left panel. The IRIS HPBW is depicted by the small circle within the rectangle drawn on the lower left corner of the right panel.

point source IRAS1758-3901. At $8.13 \mu \mathrm{m}$ a slightly extended region of intense emission can be seen southeast of RCW 122 at $(l, b)=\left(348^{\circ} .51,-1^{\circ} .11\right)$. The IRAS point source IRAS171633907 is observed projected onto this region. A large semiannular feature of low-infrared emission is observed north of RCW 122 at both frequencies. After removing the background emission, the infrared flux density of RCW 121, RCW 122, RCW $122 \mathrm{C}$, were determined for all MSX and IRAS bands. Fluxes were derived using two different methods; first, by fitting a two-dimensional Gaussian, and second, by integrating over different polygons surrounding the sources. The flux density quoted in Table 3 are average values of these two methods. The quoted uncertainty only reflects the internal consistency of our measurements.

It has been empirically found that the submillimetre spectrum of a cool dust cloud can be fitted by the product of the black-body intensity, $B_{v}\left(T_{\mathrm{d}}\right)$, and a grain emissivity that varies as $v^{m}$, where $m$ increases from $\sim 1$ at $\lambda \leq 200 \mu \mathrm{m}$ to $\geq 2$ at larger wavelengths $(\lambda \sim 1000 \mu \mathrm{m})$ (Schwartz 1982). The quoted source peak coordinates (second and third columns of Table 4) and angular extent (fourth and fifth columns) are an average of those values derived from a bidimensional Gaussian fitting to the four MSX bands. The dust temperature derived from the measured 60 and $100 \mu \mathrm{m}$ data a function of the form $v^{m} \times B_{v}\left(T_{\mathrm{d}}\right)$, adopting $m=1.0$, are given in the sixth column of Table 4 . The error quoted for $T_{\mathrm{d}}$ stems from the quoted flux density uncertainty. The IR luminosity in the $7-135 \mu \mathrm{m}$ band, seventh 
Table 3. Mid- and far-infrared flux density measurements.

\begin{tabular}{cllll}
\hline \hline $\begin{array}{c}\lambda \\
(\mu \mathrm{m})\end{array}$ & Database & $\begin{array}{l}\text { RCW 121 } \\
(\mathrm{Jy})\end{array}$ & $\begin{array}{l}\text { RCW 122 } \\
(\mathrm{Jy})\end{array}$ & $\begin{array}{l}\text { RCW 122C } \\
(\mathrm{Jy})\end{array}$ \\
\hline 8.28 & MSX-A & $226 \pm 6$ & $445 \pm 50$ & $21 \pm 2$ \\
12.00 & IRIS & $570 \pm 30$ & $810 \pm 45$ & $42 \pm 4$ \\
12.13 & MSX-C & $570 \pm 10$ & $1210 \pm 22$ & $39 \pm 4$ \\
14.65 & MSX-D & $677 \pm 7$ & $1278 \pm 17$ & $29 \pm 4$ \\
21.30 & MSX-E & $1955 \pm 50$ & $3415 \pm 260$ & $76 \pm 8$ \\
25.00 & IRIS & $2735 \pm 240$ & $5200 \pm 225$ & $147 \pm 30$ \\
60.00 & IRIS & $10950 \pm 190$ & $36300 \pm 1850$ & $2135 \pm 370$ \\
100.00 & IRIS & $12100 \pm 140$ & $33200 \pm 120$ & $4450 \pm 180$ \\
\hline
\end{tabular}

Table 4. Far-infrared parameters

\begin{tabular}{lcccclll}
\hline \hline Source & $\begin{array}{c}l \\
(\mathrm{deg})\end{array}$ & $\begin{array}{c}b \\
(\mathrm{deg})\end{array}$ & $\begin{array}{c}\Theta_{l} \\
\left({ }^{\prime}\right)\end{array}$ & $\begin{array}{l}\Theta_{\mathrm{b}} \\
\left({ }^{\prime}\right)\end{array}$ & $\begin{array}{l}T_{\mathrm{d}} \\
(\mathrm{K})\end{array}$ & $\begin{array}{l}L_{\mathrm{IR}} \\
\left(10^{5} L_{\odot}\right)\end{array}$ & $\begin{array}{l}M_{\mathrm{d}} \\
\left(M_{\odot}\right)\end{array}$ \\
\hline RCW 121 & $348^{\circ} .23$ & -0.97 & $1^{\prime} .2$ & $1^{\prime} .0$ & $46 \pm 1$ & 7.8 & $30 \pm 6$ \\
RCW 122 & $348^{\circ} .72$ & -1.05 & $1^{\prime} .5$ & 1.3 & $51 \pm 2$ & 21.0 & $62 \pm 12$ \\
RCW 122 C & $348^{\circ} .55$ & -0.98 & $0^{\prime} .6$ & 0.5 & $33 \pm 2$ & 1.5 & $40 \pm 9$ \\
\hline
\end{tabular}

column of Table 4, was calculated using the equations (Tokunaga 2000)

$F_{\mathrm{IR}}=a \times\left(b F_{12}+c F_{25}+d F_{60}+e F_{100}\right)\left(\mathrm{W} \mathrm{m}^{-2}\right)$

where $F_{i}$ is the measured flux density in the IRIS band $i$ and $a$, $b, c, d$, and $e$ are constants whose values are $1.0 \times 10^{-14}, 20.653$, $7.538,4.578$, and 1.762 , respectively, and

$L_{\mathrm{IR}}=3.112 \times 10^{7} \times d^{2} \times F_{\mathrm{IR}}\left(L_{\odot}\right)$

where $d$ is the distance in pc. Based on the flux density errors quoted in Table 3, the $F_{\text {IR }}$ values for RCW 121, RCW 122 and RCW $122 \mathrm{C}$ are accurate to within $2 \%, 3 \%$, and $9 \%$, respectively. Under the assumption that the IRIS fluxes are correctly calibrated, all $L_{\mathrm{IR}}$ estimates are accurate to within $\sim 20 \%$. The dust mass estimate from the $100 \mu \mathrm{m}$ flux, last column of Table 4 , is derived from

$M_{\mathrm{d}}=4.81 \times 10^{-12} \times F_{100} \times d^{2} \times\left[\left(\mathrm{e}^{\left(143.88 / T_{\mathrm{d}}\right.}-1\right)\right]\left(M_{\odot}\right)$

where $F_{100}$ is the flux density at $100 \mu \mathrm{m}$ in Jy; $d$ the distance in $\mathrm{pc}$; and $T_{\mathrm{d}}$ the dust temperature in $\mathrm{K}$. A mass absorption coefficient per unit length of $2.5 \mathrm{~m}^{2} \mathrm{~kg}^{-1}$ at $100 \mu \mathrm{m}$ is assumed. The dust mass uncertainty quoted in Table 4 are derived based on $F_{\text {IR }}$ uncertainties given above and $T_{\mathrm{d}}$ uncertainties given in Table 4 .

The dust temperatures and the IR luminosity derived in this paper for RCW 122 and RCW 122 C (see Table 4), are in good agreement with those values derived by Ghosh et al. (1989) for the same sources, namely $\sim 60 \mathrm{~K}$ and $2.8 \times 10^{6} L_{\odot}$ (RCW 122) and $\sim 35 \mathrm{~K}$ and $1.5 \times 10^{5} L_{\odot}(\mathrm{RCW} 121)$. Based on the molecular gas masses quoted in Table 1 and the dust masses given in Table 4, the gas-to-dust ratio varies between a minimum of $\sim 1150$ (RCW 121) to a maximum of $\sim 3550$ (RCW 122). The weighted average of this ratio is $\sim 1500 \pm 400$. On the average this ratio appears to be a factor $\sim 11 \pm 5$ higher than the value usually assumed for the inner Galaxy, $140 \pm 50$ (Tokunaga 2000). Nonetheless, the large uncertainty of the derived gas-todust ratio casts doubt on the overabundance of dust in these RCW sources.

Figure 9 shows that the bulk of the IR emission (82\% for RCW 121, 90\% for RCW 122, and 95\% for RCW 122 C) arises from grains emitting at $\lambda \geq 25 \mu \mathrm{m}$. Figure 9 also shows that the dust grains having the dust temperature given in Table 4
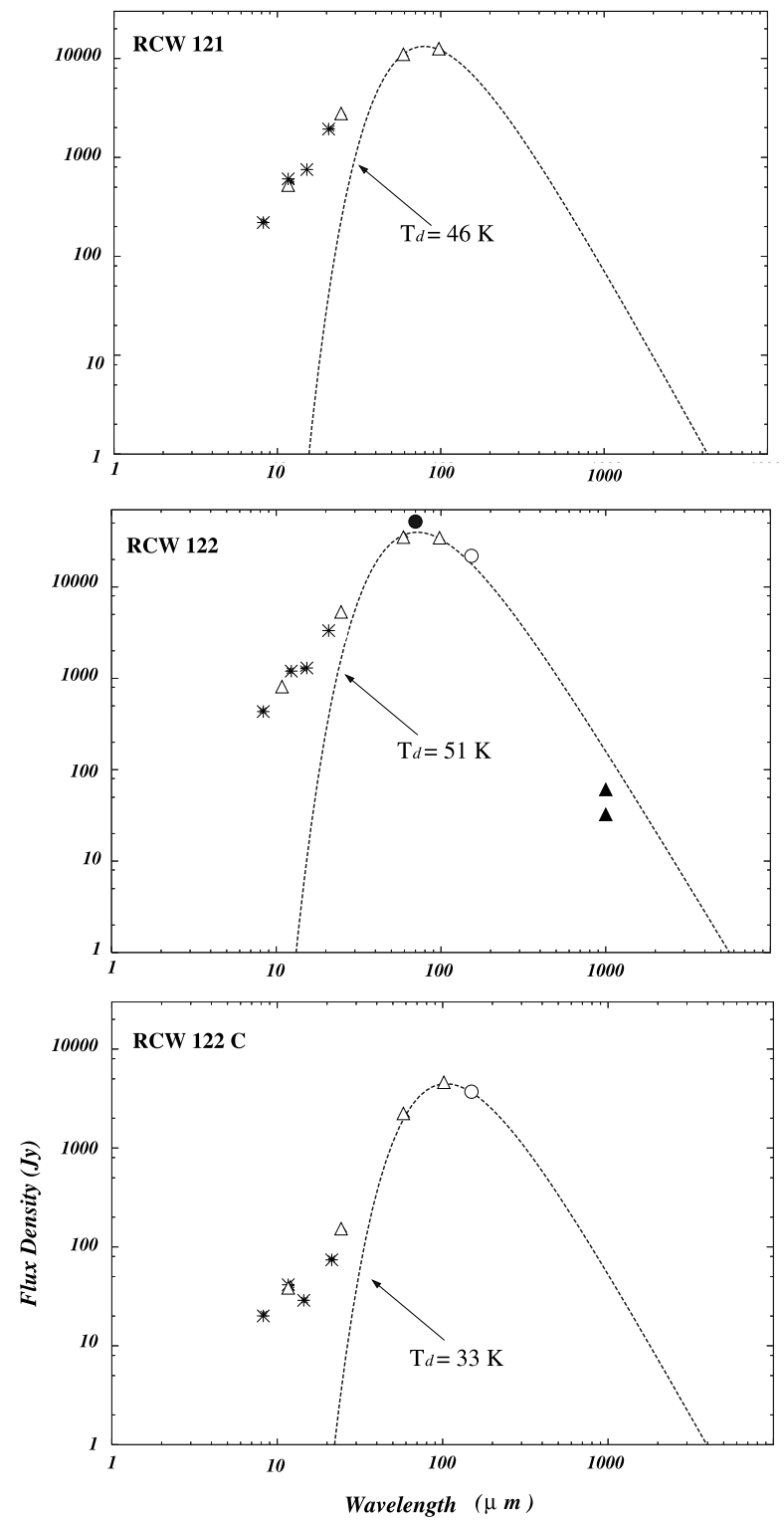

Fig. 9. Mid- and far-infrared spectral energy distribution of RCW 121 (upper panel), RCW 122 (middle panel), and RCW 122 C (lower panel). The asterisks and triangles denote the MSX and IRIS measurements, respectively. The ballon-borne measurement of Ghosh et al. (1989) at $151 \mu \mathrm{m}$ is marked by an open circle, the $69 \mu \mathrm{m}$ measurement of McBreen et al. (1985) is signaled by a filled circle, and the IR flux density at $300 \mathrm{GHz}$ (see text) is indicated by a filled triangle. Dashed lines are emission of blackbodies with a temperature $T_{\mathrm{d}}$ with a $\lambda^{-1}$ emissivity law.

falls short of the MSX and IRIS measurements at shorter wavelengths. Though emission lines arising from atoms present in the gaseous phase and/or from polycyclic aromatic hydrocarbons (PAHs) may contribute to the IR fluxes measured at shorter wavelengths, the emission at shorter wavelengths is believed to be due to very small grains heated by stellar UV photons or by collisions with energetic electrons (Puget \& Léger 1989). The RCW 122 far-infrared emission at $300 \mathrm{GHz}$ is included in the middle panel of Fig. 9. The IR emission at this frequency is derived by subtracting from the observed flux density the extrapolated free-free continum flux density at that frequency. The latter turns out to be $21.7 \pm 2.8 \mathrm{Jy}$, using our $5 \mathrm{GHz}$ flux density measurement and a spectral index of $-0.14 \pm 0.03$. 


\section{Conclusions}

The ${ }^{12} \mathrm{CO}$ molecular gas distribution within a square region $1.47 \times 1^{\circ} .45$ in size centred at $(l, b)=\left(348^{\circ} .52,-1^{\circ} .055\right)$ has been analysed to investigate the relationship among the Wolf-Rayet stars WR 87, WR 89, WR 91, the open cluster HM 1 and the star forming regions RCW 122, RCW $122 \mathrm{C}$, and RCW 121.

Relying on the morphological correlation between the infrared emission depicted by the MSX Band A image and the CO molecular emission in the velocity range from -18 to $-5 \mathrm{~km} \mathrm{~s}^{-1}$, a giant molecular complex (GMC) is revealed for the first time associated with the star forming regions RCW 121 and RCW 122 (A, B and C). Adopting a distance of $5 \mathrm{kpc}$ (Radhakrishnan et al. 1972), the GMC has a mean linear size $(\Delta l \times \Delta b)$ of $\sim 100 \times 20$ pc. Between $348^{\circ} 55 \leq l \leq 348^{\circ}$. 8 , the GMC extends further to the south reaching a galactic latitude $b \sim-1.6$. Within the above galactic longitude interval, the GMC stretches in galactic latitude by almost $50 \mathrm{pc}$.

The total mass of molecular gas is $\sim 1.2 \times 10^{6} M_{\odot}$, and its mean volume molecular hydrogen density is about $120 \mathrm{~cm}^{-3}$. In the same velocity range, -18 to $-5 \mathrm{~km} \mathrm{~s}^{-1}$, where the strong emission of the molecular gas associated with the three RCWs is easily noticeable, there is patchy and weak $\mathrm{CO}$ emission towards the area where WR 87, WR 89, and HM 1 are located. This fact may imply that the northern part of the IR ring-like feature, whose geometric centre falls in the vicinity of HM 1, may be unrelated to the GMC. This conclusion may be in line with the different distances found for HM 1 (2.9 kpc, Mermilliod 1999) and for RCW 121 and RCW 122 (5.0 kpc, Radhakrisnan et al. 1972).

Within the GMC, the regions RCW 122, RCW $122 \mathrm{C}$ and RCW 121 are related to well-defined CO concentrations. The individual mass of these concentrations range from $2.2 \times 10^{5} M_{\odot}$ (RCW 122) to $\sim 0.5 \times 10^{5} M_{\odot}(\mathrm{RCW} 122 \mathrm{C})$. The linear dimensions of these concentrations range from $30 \times 21 \mathrm{pc}(\mathrm{RCW} 122)$ to $24 \times 11 \mathrm{pc}(\mathrm{RCW} 122 \mathrm{C})$. The CO lines observed towards these concentrations are broad, typically between 5 to $7 \mathrm{~km} \mathrm{~s}^{-1}$, and the individual CO profiles show clear evidence of a complex dynamics, illustrated by the presence of line asymmetries and/or double peaks.

Based on the correlation between the IR emission and the $\mathrm{CO}$ distribution, the similarity of the radial velocity among the three main molecular concentrations, and the lack of $\mathrm{CO}$ emission in the velocity range -18 to $-5 \mathrm{~km} \mathrm{~s}^{-1}$ related to the arclike IR feature located north of RCW 122, it is concluded that RCW 121, RCW 122, and RCW $122 \mathrm{C}$ are very likely different star forming regions within the same molecular complex.

New flux density determinations, derived from already existing databases confirm the thermal nature of both RCW 122 and RCW 121. The radio continuum spectrum of RCW 122 at high frequencies $(v \sim 300 \mathrm{GHz})$ shows the typical bump of infrared emission above the expected level of pure free-free emission. On the other hand, at low frequencies $(v \sim 408 \mathrm{MHz})$ the observed flux density indicates that this source may be optically thick. The radio continuum spectrum of this source derived under the assumption of an $\mathrm{EM}=2.3 \times 10^{5} \mathrm{pc} \mathrm{cm}^{-6}$ (this paper) and an electron temperature of $7150 \mathrm{~K}$ (Quireza et al. 2006b) is consistent with this interpretation.

In the case of RCW 121, the number of continuum flux density measurements have been increased by a factor of 3 by our new estimates. Unlike RCW 122, this source does not show evidence of being optically thick at low frequencies. According to our analysis, the continuum spectrum of RCW 121 is consistent with an emission measure of $\mathrm{EM} \simeq 1.7 \times 10^{4} \mathrm{pc} \mathrm{cm}^{-6}$, an electron density of $n_{\mathrm{e}} \simeq 170 \mathrm{~cm}^{-3}$, and a turnover frequency of $220 \pm 20 \mathrm{MHz}$. Our EM estimate is almost an order of magnitude lower than the one quoted by Roman-Lopes \& Abraham (2006). Radio continuum measurements at lower frequencies (e.g., the $843 \mathrm{MHz}$ Sydney University Molonglo Sky Survey (SUMSS, Murphy et al. 2007, continuum survey) may be useful to settle this discrepancy. The high EM value of Roman-Lopes \& Abraham would be compatible with $843 \mathrm{MHz}$ continuum flux density falling in the range 6 to $7 \mathrm{Jy}$, while our low EM value would be more in line with a higher radio continuum flux at $843 \mathrm{MHz}$, namely around $12 \mathrm{Jy}$. Based on the number of ionizing photons required to maintain the ionization level of these HII regions, several O-type stars are needed to power each source.

The three RCWs regions have strong mid- and far-infrared counterparts. These sources are stronger, by at least two orders of magnitude, at longer $(\lambda \geq 60 \mu \mathrm{m})$ than at shorter $(\lambda \leq 25 \mu \mathrm{m})$ wavelengths. The dust temperature of the grains responsible of the far-infrared emission varies between a minimum of $33 \mathrm{~K}$ (RCW 121) to a maximum of $51 \mathrm{~K}$ (RCW 122). The luminosity in the 7 to $135 \mu \mathrm{m}$ band varies between $1.5 \times 10^{5} L_{\odot}(\mathrm{RCW} 121)$ and $2.1 \times 10^{6} L_{\odot}(\mathrm{RCW} 122)$.

Acknowledgements. This work was partially supported by the Agencia Nacional de Promoción Científica y Tecnológica (ANPCyT) under project PICT $14018 / 03$. This research has made use of the WEBDA database, operated at the Institute for Astronomy of the University of Vienna. We greatly appreciate the hospitality of all staff members of Las Campanas Observatory of the Carnegie Institute of Washington. We thank all members of the NANTEN staff, in particular, Prof. Yasuo Fukui, Dr. Toshikazu Onishi, Dr. Akira Mizuno, and students Y. Moriguchi, H. Saito, and S. Sakamoto. We would like to thank the anonymous referee for her/his helpful suggestions. We also would like to thank Dr. D. Miniti (Pontificia Universidad Católica, Chile) and Mr. F. Bareilles (IAR) for their involvement in early stages of this project.

\section{References}

Arnold, E. M., Kreysa, E., Schultz, G. V., \& Sherwood, W. A. 1978, A\&A, 70, L1

Baars, J. W. M., Genzel, R., Pauliny-Toth, I. I. K., \& Witzel, A. 1977, A\&A, 61, 99

Batchelor, R. A., Caswell, J. L., Goss, W. M., et al. 1980, Aust. J. Phys., 33, 139 Batchelor, R. A., McCulloch, M. G., \& Whiteoak, J. B. 1981, MNRAS, 194, 911 Beard, M., Maca Thomas, B., \& Day, G. A. 1969, Aust. J. Phys. Astroph. Suppl., N11, 27

Bohlin, R. C., Savage, B. D., \& Drake, J. F. 1978, ApJ, 224, 132

Brand, J., van der Bij, M. D. P., de Vries, C.P., et al. 1984, A\&A, 139, 181

Bronfmann, L., Nyman, L.-Å., \& May, J. 1996, A\&AS, 115, 81

Brown, R. L., \& Broderick, J. J. 1973, ApJ, 181, 125

Cappa, C. E. 2006, RevMEXAA(SC), 26, 9

Caswell, J. L. 1997, MNRAS, 289, 203

Caswell, J. L. 2003, MNRAS, 341, 551

Caswell, J. L., \& Haynes, R. F. 1987, A\&A, 171, 261

Caswell, J. L., \& Robinson, B. J. 1974, Aust. J. Phys., 27, 597

Caswell, J. L., Batchelor, R. A., Forster, R. A., \& Wellington, K. J. 1983, Aust. J. Phys., 36, 401

Caswell, J. L., Vaile, R. A., Ellingsen, S. P., \& Norris, R. P. 1995, MNRAS, 274, 1126

Cheung, L. H., Frogel, J. A., Gezari, D. Y., \& Hauser, M. G. 1980, ApJ, 240, 74 Dias, W. S., Alessi, B. S., Moitinho, A., \& Lepine, J. R. D. 2002, A\&A, 389, 171 Digel, S. W., Lyder, D. A., Philbrick, A. J., Puche, D., \& Thaddeus, P. 1996, ApJ, 458,561

Duncan, A. R., Steward, R. J., Haynes, R. F., \& Jones, K. L. 1995, MNRAS, 277, 36

Emerson, J. P., Jennings, R. E., \& Moorwood, A. F. M. 1973, ApJ, 184, 401

Gardner, F. F., \& Whiteoak, J. B. 1978, MNRAS, 183, 711

Gaume, R. A., \& Mutel, R. L. 1987, ApJS, 65, 193

Ghosh, S. K., Iyengar, K. V. K., Rengarajan, T. N., et al. 1989, ApJS, 69, 233

Gillespie, A. R., White, G. J., \& Watt, G. D. 1979, MNRAS, 186, 383

Goss, W. M., \& Shaver, P. A. 1970, Aust. J. Phys. Suppl., 14, 1

Goss, W. M., Murray, J. D., \& Radhakrishnan, V. 1970, Proc. ASA, 1, 332

Haslam, C. G. T. 1974, A\&AS, 15, 333

Haynes, R. F., Caswell, J. L., \& Simons, L. W. J. 1978, Aust. J. Phys. Suppl., 45,1

Juvela, M. 1996, A\&AS, 118, 191 
Kaufmann, P., Gammon, R. H., Ibanez, A. L., et al. 1976, Nature, 260, 306 Kutner, M. L., \& Ulich, B. L. 1981, ApJ, 250, 341

Langston, G., Minter, A., D'Addario, L., et al. 2000, AJ, 119, 2801

Martin, R. N., Emerson, D. T., Ruf, K., Wilson, T. L., \& Zimmermann, P. 1983, in Surveys of the Southern Galaxy, ed. W. B. Burton, \& F. P. Israel (Dordrecht: Reidel), 217

McBreen, B., Fazio, G. G., Loughran, L., \& Rengarajan, T. N. 1985, AJ, 90, 88 McGee, R. X., \& Newton, L. M. 1981, MNRAS, 196, 889

Mermilliod, J.-C. 1999, WEBDA database, http: //obswww . unige.ch/webda Miville-Deschênes, M. A., \& Lagache, G. 2005, ApJS, 157, 302

Moriguchi, Y., Yamaguchi, N., Onishi, T., Mizuno, A., \& Fukui, Y. 2001, PASJ, 53,1025

Murphy, Y., Mauch, T., Green, A., et al. 2007 [arXiv: 0708. 3092]

Penzias, A. A., \& Burrus, C. A. 1973, ARA\&A, 11, 51

Price, S. D., Egan, M. P., Carey, S., Mizuno, D., \& Kuchar, T. 2001, AJ, 121, 2819

Puget, J. L., \& Léger, A. 1989, ARA\&A, 27, 161

Quireza, C., Rood, R. T., Balsan, D. S., \& Bania, T. M. 2006a, ApJS, 165, 338

Quireza, C., Rood, R. T., Bania, T. M., et al. 2006b, ApJ, 653, 1226

Radhakrishnan, V., Goss, W. M., Murray, J. D., \& Brooks, J. W. 1972, ApJS, 24, 49

Rodgers, A. W., Campbell, C. T., \& Whiteoak, J. B. 1960, MNRAS, 121, 103

Roman-Lopez, A., \& Abraham, Z. 2006, AJ, 131, 951

Sanduleak, N. 1974, PASP, 86, 461

Schaerer, D., \& de Koter, A. 1997, A\&A, 322, 598
Schraml, J., \& Mezger, P. G. 1969, ApJ, 156, 269

Schwartz, P. R. 1982, ApJ, 252, 589

Shaver, P. A., \& Goss, W. M. 1970a, Aust. J. Phys. Suppl., 14, 77

Shaver, P. A., \& Goss, W. M. 1970b, Aust. J. Phys. Suppl., 14, 133

Shaver, P. A., McGee, R. X., Newton, L. M., Danks, A. C., \& Pottasch, S. R. 1983, MNRAS, 204, 53

Simpson, J. P., \& Rubin, R. H. 1990, ApJ, 354, 165

Sollins, P. K., \& Megeath, S. T. 2004, AJ, 128, 2387

Tokunaga, A. T. 2000, in Allen's Astrophysical Quantities, Fourth Edition, ed. A. N. Cox (Springer), 143

Ulich, B. L., \& Hass, R. W. 1976, ApJS, 30, 247

van der Hucht, K. A. 2001, New Astron. Rev., 45, 135

Walsh, A. J., Hyland, A. R., Robinson, G., \& Burton, M. G. 1997, MNRAS, 291, 261

Walsh, A. J., Burton, M. G., Hyland, A. R., \& Robinson, G. 1998, MNRAS, 301, 640

Whiteoak, J. B., \& Gardner, F. F. 1974, A\&A, 37, 389

Whiteoak, J. B., \& Gardner, F. F. 1978, MNRAS, 185, 33P

Whiteoak, J. B., Otrupcek, R. E., \& Rennie, C. J. 1982, Proc. ASA, 4, 434

Wilson, T. L., Mezger, P. G., Gardner, F. F., \& Milne, D. K. 1970, A\&A, 6, 364

Whitworth, A. S., Bhattal, A. S., Chapman, M. J., Disney, M. J., \& Turner, J. A. 1994, MNRAS, 268, 291

Wright, A. E., Griffith, M. R., Burke, B. E., \& Ekers, R. D. 1994, ApJS, 91, 111

Zhang, C. Y., Harvey, P. M., Smith, B. J., Colomé, C., \& DiFrancesco, J. 1995, ApJ, 452, 308 